\title{
THE 2MASS REDSHIFT SURVEY—DESCRIPTION AND DATA RELEASE
}

\author{
John P. Huchra ${ }^{1,15}$, Lucas M. Macri ${ }^{2}$, Karen L. Masters ${ }^{3,4}{ }^{\text {, }}$ Thomas H. Jarrett $^{5}$, Perry Berlind ${ }^{1}$, \\ Michael Calkins ${ }^{1}$, Aidan C. Crook $^{6}$, Roc Cutri ${ }^{4}$, Pirin ErdoǦdu ${ }^{7}$, Emilio Falco ${ }^{1}$, Teddy George ${ }^{8}$, \\ Conrad M. Hutcheson ${ }^{9}$, Ofer Lahav ${ }^{7}$, JefF Mader ${ }^{10}$, Jessica D. Mink ${ }^{1}$, Nathalie Martimbeau ${ }^{11}$, \\ Stephen Schneider $^{12}$, Michael Skrutskie ${ }^{13}$, Susan ToKarz ${ }^{1}$, and Michael Westover ${ }^{14}$ \\ ${ }^{1}$ Harvard-Smithsonian Center for Astrophysics, 60 Garden Street, Cambridge, MA 02138, USA \\ ${ }^{2}$ George P. and Cynthia Woods Mitchell Institute for Fundamental Physics and Astronomy, Department of Physics and Astronomy, \\ Texas A\&M University, 4242 TAMU, College Station, TX 77843, USA; 1macri@tamu.edu \\ ${ }^{3}$ Institute for Cosmology and Gravitation, University of Portsmouth, Dennis Sciama Building, Burnaby Road, \\ Portsmouth, PO1 3FX, UK; karen.masters@ port.ac.uk \\ ${ }^{4}$ SEPNet (South East Physics Network), UK \\ ${ }^{5}$ Infrared Processing and Analysis Center, California Institute of Technology, 770 S Wilson Ave., Pasadena, CA 91125, USA \\ ${ }^{6}$ Microsoft Corp., 1 Microsoft Way, Redmond, WA 98052, USA \\ ${ }^{7}$ Department of Physics and Astronomy, University College London, London WC1E 6BT, UK \\ ${ }^{8}$ Canada-France-Hawaii Telescope, 65-1238 Mamalahoa Hwy, Kamuela, HI 96743, USA \\ ${ }^{9}$ Kavli Institute for Particle Astrophysics and Cosmology, Stanford University, Stanford, CA 94309, USA \\ ${ }^{10}$ Keck Observatory, 65-1120 Mamalahoa Hwy, Kamuela, HI 96743, USA \\ ${ }^{11}$ Planétarium de Montréal, 1000 rue Saint-Jacques, Montréal, Québec H3C 1G7, Canada \\ 12 Department of Astronomy, University of Massachusetts, Amherst, MA 01003, USA \\ ${ }^{13}$ Department of Astronomy, University of Virginia, Charlottesville, VA 22904, USA \\ ${ }^{14}$ McKinsey \& Co., 1420 Fifth Ave., Ste 3100, Seattle, WA 98101, USA \\ Received 2011 July 29; accepted 2011 December 17; published 2012 March 14
}

\begin{abstract}
We present the results of the 2MASS Redshift Survey (2MRS), a ten-year project to map the full three-dimensional distribution of galaxies in the nearby universe. The Two Micron All Sky Survey (2MASS) was completed in 2003 and its final data products, including an extended source catalog (XSC), are available online. The 2MASS XSC contains nearly a million galaxies with $K_{s} \leqslant 13.5 \mathrm{mag}$ and is essentially complete and mostly unaffected by interstellar extinction and stellar confusion down to a galactic latitude of $|b|=5^{\circ}$ for bright galaxies. Near-infrared wavelengths are sensitive to the old stellar populations that dominate galaxy masses, making 2MASS an excellent starting point to study the distribution of matter in the nearby universe. We selected a sample of 44,599 2MASS galaxies with $K_{s} \leqslant 11.75 \mathrm{mag}$ and $|b| \geqslant 5^{\circ}\left(\geqslant 8^{\circ}\right.$ toward the Galactic bulge) as the input catalog for our survey. We obtained spectroscopic observations for 11,000 galaxies and used previously obtained velocities for the remainder of the sample to generate a redshift catalog that is $97.6 \%$ complete to well-defined limits and covers $91 \%$ of the sky. This provides an unprecedented census of galaxy (baryonic mass) concentrations within $300 \mathrm{Mpc}$. Earlier versions of our survey have been used in a number of publications that have studied the bulk motion of the Local Group, mapped the density and peculiar velocity fields out to $50 h^{-1} \mathrm{Mpc}$, detected galaxy groups, and estimated the values of several cosmological parameters. Additionally, we present morphological types for a nearly complete sub-sample of 20,860 galaxies with $K_{s} \leqslant 11.25$ mag and $|b| \geqslant 10^{\circ}$.
\end{abstract}

Key words: catalogs - galaxies: distances and redshifts - surveys

Online-only material: color figures, machine-readable tables

\section{INTRODUCTION}

Between the mid-1970s and the early 1980s, several discoveries were made based on innovations in detector technology and better understanding of galaxies that substantially changed our view of the nearby universe. The cosmic microwave background (CMB) dipole was convincingly measured (Corey \& Wilkinson 1976; Smoot et al. 1977; Cheng et al. 1979), the first large redshift surveys were begun (cf. Davis et al. 1982), and Virgo Infall was both convincingly predicted and measured (de Vaucouleurs 1956; Silk 1974; Peebles 1976; Aaronson et al. 1982). The kinematics of the Local Universe became a cosmological test and tool, and-with the realization that the Virgo supercluster was insufficient to explain the CMB dipole-the search for the source of the flow (astronomy's Nile) became a major cosmological quest.

\footnotetext{
${ }^{15}$ This paper is mostly based on the text written by John Huchra before his death in 2010 October.
}

In the 1980s, this quest led to the discovery of even larger mass concentrations such as the Great Attractor (Burstein et al. 1986; Lynden-Bell et al. 1988) and the Shapley Supercluster (Tully \& Shaya 1984; Tammann \& Sandage 1985), and the initiation of several very large scale redshift surveys based on IR and optical catalogs (e.g., Strauss et al. 1992; Santiago et al. 1995; Saunders et al. 2000). Perforce then followed advanced distance surveys and catalogs (Mould et al. 1993; Willick et al. 1997). Sophisticated techniques were developed to analyze these surveys (Dekel et al. 1990; Zaroubi et al. 1995), but despite reasonable data and thorough analyses, the source of the $\mathrm{CMB}$ dipole was not convincingly identified and there remained very significant conflicts between the results of different surveys (e.g., Schmoldt et al. 1999).

Near the end of the 1990s, a conflict remained between $\Omega_{M}$ on all measured scales and the $\Omega_{M}=1$ strongly predicted from inflation and cold dark matter models. Was the discrepancy real or were there problems with the data and/or the theory? Most of the community realized that all extant maps were tremendously 
biased, either by extinction or by wavelength (read "young star formation," which dominates both blue and far-infrared light). This was the explanation advocated by the theorists- the galaxies being measured were not really tracing the mass.

Fortunately, the overall $\Omega$ problem was solved soon thereafter with the discovery of dark energy (Riess et al. 1998; Perlmutter et al. 1999) coupled with the accurate determination of the Hubble constant (Freedman et al. 2001) and the measurement of the large-scale geometry of the universe through observations of fluctuations in the CMB (Spergel et al. 2003). Still, several very significant questions remain. Can we accurately (to a few percent) observationally account for the matter density in the nearby universe? How is matter distributed? In particular, can we explain gravitationally the motion of the Milky Way with respect to the CMB? Do we understand the differences, if any, in the distribution of ordinary baryonic matter and dark matter (i.e., the bias function)? These questions are yet unanswered and clearly drive the detailed understanding of galaxy and largescale structure formation and evolution.

Despite all of the aforementioned work, even the galaxy density field of the Local Supercluster (LSC) is not in good shape. Despite high-quality data on the flow field, Tonry et al. (2000) found there are many missing elements to the model of the LSC, including possible local sources of the observed quadrupole field and the "Local Anomaly."

\section{THE TWO MICRON ALL SKY SURVEY}

The Two Micron All Sky Survey (2MASS; Skrutskie et al. 2006) had its origins in a proposal to NASA for a "Near InfraRed Astronomical Satellite" by G. Fazio, J. Huchra, J. Mould, and collaborators in 1988. The survey was eventually carried out by a team led by astronomers at the University of Massachusetts (UMass) using twin $1.3 \mathrm{~m}$ telescopes located at Mount Hopkins, AZ (starting in 1997) and Cerro Tololo, Chile (starting in 1998). Scans were completed by 2001 and the final data release was made available in 2003 through IPAC. ${ }^{16}$

2MASS mapped the entire sky in the $J, H$, and $K_{s}$ bands, avoiding many of the observational biases that affected previous optical and far-infrared all-sky surveys. The effects of interstellar extinction are reduced by $10 \times$ relative to the $B$ band and the spectral energy distributions of most galaxies peak at near-infrared wavelengths. Moreover, $K$-band luminosities are a useful proxy for baryonic mass as the stellar mass-to-light ratio is fairly constant across galaxy types at this wavelength (e.g., within a factor of two; Bell \& de Jong 2001). This makes the near-infrared the spectral region of choice to map the distribution of matter in the nearby universe.

The 2MASS photometric pipeline produced a complete and reliable extended source catalog (XSC; Jarrett et al. 2000; Jarrett 2004) of $\sim 10^{6}$ objects with $K_{s} \leqslant 13.5 \mathrm{mag}$ and a mean photometric accuracy better than $0.1 \mathrm{mag}$. Moreover, the database included information on the photometric structure of the galaxies (photometric profiles, axis ratios, etc.). 2MASS provided the first modern, all-sky, highly accurate catalog of galaxies. A few years later, the Sloan Digital Sky Survey (SDSS; York et al. 2000) started to provide overlapping deeper optical data which eventually covered $\sim 35 \%$ of the sky (Aihara et al. 2011), but 2MASS remains the only modern survey which can be used to construct a uniform, all-sky, three-dimensional map of the Local Universe.

\footnotetext{
16 http://www.ipac.caltech.edu/2mass/
}

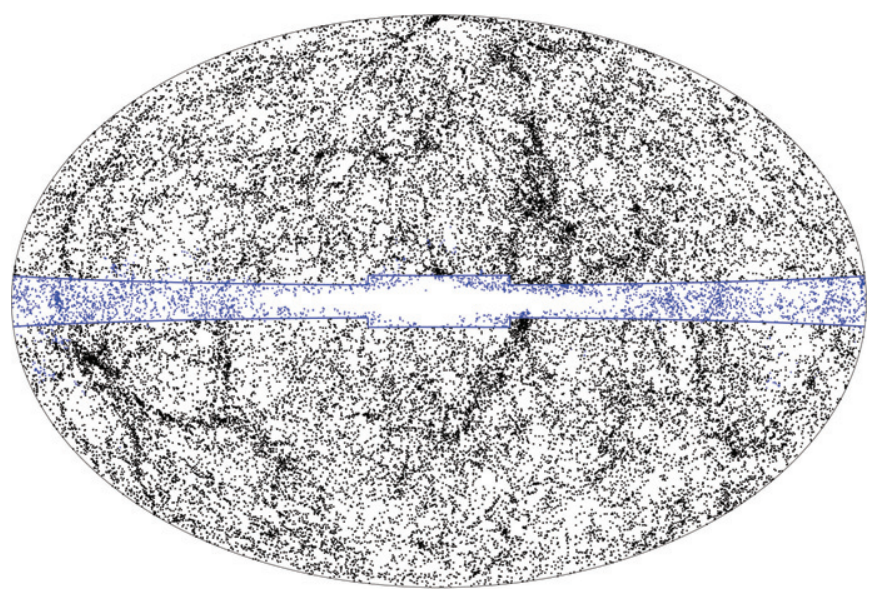

Figure 1. Distribution of 2MASS galaxies with $K_{s} \leqslant 11.75$ mag in Galactic coordinates (Aitoff projection). Blue dots represent galaxies outside our survey area. Note that due to stellar confusion we cannot cover, even to this bright magnitude limit, the very central region of the galaxy, but we do cover $\sim 91 \%$ of the sky.

(A color version of this figure is available in the online journal.)

Two decades before 2MASS, the first flux-limited all-sky galaxy catalog was created from observations by the IRAS satellite at $60 \mu \mathrm{m}$ (Strauss et al. 1990). Since galaxies were unresolved by IRAS, the point source catalog formed the basis of a redshift survey (PSCz; Fisher et al. 1995; Saunders et al. 2000). Among other problems, the PSCz catalog gave little weight to ellipticals (which are dim at $60 \mu \mathrm{m}$ because this wavelength is dominated by dusty star formation) and suffered from severe confusion in regions of high density. However, the uniform fullsky coverage was unique at the time.

\subsection{The Zone of Avoidance}

2MASS is an excellent probe of the zone of avoidance for bright galaxies, as was discussed in depth by Huchra et al. (2005). Figure 1 is an updated version of Figure 8 from Huchra et al. (2005) showing the 2MASS XSC coverage at $K_{s} \leqslant 11.75 \mathrm{mag}$, limited only by confusion near the galactic center. Figure 2 is an updated version of Figure 7 from Huchra et al. (2005) and shows the galaxy surface density versus galactic latitude for several magnitude limits. At the bright magnitudes surveyed by $2 \mathrm{MRS}$, the catalog is essentially complete to very low latitudes.

\section{THE 2MASS REDSHIFT SURVEY}

The primary extragalactic goal of 2MASS was to feed the next generation of all-sky redshift surveys to fully map the nearby universe. To this end, we started a program in 1997 September to obtain the required spectroscopic data for a magnitude-limited sample of galaxies: the 2MASS Redshift Survey (2MRS). Our initial survey limits of $K_{s}=11.25 \mathrm{mag}$ and $|b|=10^{\circ}(20,860$ galaxies; hereafter 2MRS11.25) were progressively increased to final values of $K_{s}=11.75 \mathrm{mag}$ and $|b|=5^{\circ}-8^{\circ}(44,599$ galaxies; the full 2MRS), allowing us to steadily complete our view of the Local Universe.

2MRS builds and improves on the previous generation of local surveys (see Table 1) and is complementary to contemporaneous larger, deeper surveys, notably $2 \mathrm{dF}$ (Colless et al. 2001), SDSS (Aihara et al. 2011), and specially 6dFGS (Jones et al. 2004, 2005, 2009) which also used the 2MASS XSC as its input catalog and provided a large number of redshifts for our survey. 
Table 1

Large Redshift Surveys of the Nearby Universe to Date

\begin{tabular}{|c|c|c|c|c|c|}
\hline Survey & $\begin{array}{l}\text { Sky Coverage } \\
(\% 4 \pi \mathrm{sr})\end{array}$ & $\begin{array}{l}\text { Depth }^{\mathrm{a}} \\
\quad(z)\end{array}$ & $\begin{array}{l}\text { Selection } \\
\text { (band, flux) }\end{array}$ & $\begin{array}{l}\text { No. of Gals. } \\
\qquad\left(\times 10^{3}\right)\end{array}$ & Reference \\
\hline CfA1 & $30 \%$ & 0.03 & $B=14.5 \mathrm{mag}$ & 2.4 & de Lapparent et al. (1986) \\
\hline ORS & $60 \%$ & 0.03 & $B=14.0 \mathrm{mag}$ & 8.5 & Santiago et al. (1995) \\
\hline $\begin{array}{l}\text { SSRS2+ } \\
\text { CfA2 }\end{array}$ & $60 \%$ & 0.04 & $B=15.5 \mathrm{mag}$ & 23.6 & $\begin{array}{l}\text { da Costa et al. (1998) and } \\
\text { Huchra et al. (1999) }\end{array}$ \\
\hline IRAS PSCz & $85 \%$ & 0.08 & $60 \mu \mathrm{m}=0.6 \mathrm{Jy}$ & 16.1 & Saunders et al. (2000) \\
\hline LCRS & $1 \%$ & 0.17 & $R=17.5 \mathrm{mag}$ & 25.3 & Shectman et al. (1996) \\
\hline $2 \mathrm{dF}$ & $8 \%$ & 0.19 & $b_{\mathrm{J}}=19.5 \mathrm{mag}$ & 245.6 & Colless et al. (2001) \\
\hline $\mathrm{SDSS}^{\mathrm{b}}$ & $35 \%$ & 0.33 & $r=17.5 \mathrm{mag}$ & 943.6 & Aihara et al. (2011) \\
\hline 6dFGS & $40 \%$ & 0.10 & $K_{s}=12.65 \mathrm{mag}$ & 124.6 & Jones et al. $(2004,2005,2009)$ \\
\hline 2MRS11.25 & $83 \%$ & 0.04 & $K_{s}=11.25 \mathrm{mag}$ & 20.6 & Huchra et al. (2005) \\
\hline 2MRS & $91 \%$ & 0.05 & $K_{s}=11.75 \mathrm{mag}$ & 43.5 & This work \\
\hline
\end{tabular}

Notes.

a 90 percentile redshift value in catalog.

b DR8 main galaxy sample.

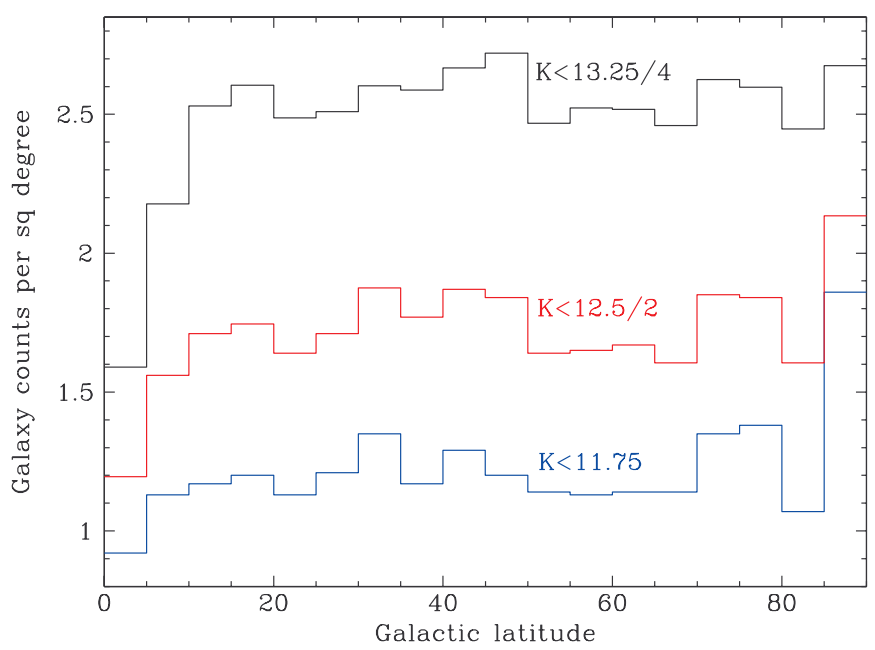

Figure 2. Surface number density vs. galactic latitude for three cuts in the 2MASS XSC at $K_{s}=11.75,12.5$, and $13.25 \mathrm{mag}$. While the number counts drop sharply in the $5^{\circ} \leqslant|b| \leqslant 10^{\circ}$ bin for the 13.25 mag sample, the incompleteness is reduced for the $12.5 \mathrm{mag}$ sample and it is essentially zero for the $11.75 \mathrm{mag}$ sample. The upturn in all samples at $90^{\circ}$ is due to the Coma supercluster.

(A color version of this figure is available in the online journal.)

These larger surveys have not attempted to be complete over the whole sky, since many cosmological measurements do not require this level of completeness and tradeoffs must be made between depth and sky coverage given available telescope time and resources.

\subsection{Sample Selection}

The initial selection of sources was based on the 2MASS XSC. The 2MASS photometric pipeline performed a variety of magnitude measurements for each extended source in each band. We selected as our primary set of magnitudes the isophotal magnitudes measured in an elliptical aperture defined at the $K_{s}=20 \mathrm{mag} / \square^{\prime \prime}$ isophote. We also include in our data tables the "total extrapolated magnitudes" derived by the pipeline, but do not use them for our sample selection. In the case of galaxies with angular sizes much greater than the width of a single 2MASS scan, we used the photometry presented in the 2MASS Large Galaxy Atlas (LGA) by Jarrett et al. (2003). We applied a modest extinction correction to the 2MASS XSC or LGA magnitudes using the maps of Schlegel et al. (1998).

We selected 45,086 sources which met the following criteria.

1. $K_{s} \leqslant 11.75 \mathrm{mag}$ and detected at $H$.

2. $E(B-V) \leqslant 1 \mathrm{mag}$.

3. $|b| \geqslant 5^{\circ}$ for $30^{\circ} \leqslant l \leqslant 330^{\circ} ;|b| \geqslant 8^{\circ}$ otherwise.

We rejected 324 sources of galactic origin (multiple stars, planetary nebulae, and $\mathrm{H}$ II regions) or pieces of galaxies detected as separate sources by the 2MASS pipeline. Additionally, we flagged 314 bona fide galaxies with compromised photometry for reprocessing at a future date. Some of these galaxies have bright stars very close to their nuclei which were not detected by the pipeline. Others are in regions of high stellar density and their center positions and/or isophotal radii have been incorrectly measured by the pipeline. Lastly, some are close pairs or multiples but the pipeline only identified a single object. A detailed explanation of the steps taken to reject and reprocess the flagged galaxies is given in the Appendix.

In summary, the final input catalog contains 44,599 entries which are plotted using black symbols in Figure 1. Galaxies outside the survey area are plotted in blue and outline the "zone of avoidance" described previously. In this work, we present redshifts for 43,533 of the selected galaxies, or $97.6 \%$ of the sample.

\subsection{Observations, Data Reduction, and Analysis}

We obtained spectra for 11,000 galaxies that met the selection criteria listed above, plus an additional 2,898 galaxies beyond the catalog limits. Observations were carried out between 1997 September and 2011 January using a variety of facilities which are listed in Table 2. The majority of the spectra obtained for this survey were acquired at the Fred L. Whipple Observatory (FLWO) $1.5 \mathrm{~m}$ telescope, which mostly targeted galaxies in the northern hemisphere. In the southern hemisphere, we relied heavily on observations by the 6dFGS project (Jones et al. 2004, 2005, 2009) but also carried out our own observations using the Cerro Tololo Interamerican Observatory (CTIO) 1.5 m telescope. We initially targeted $K_{s}<11.25$ mag galaxies to obtain a complete all-sky sample (Huchra et al. 2005) while 6dFGS observations were still ongoing. Later, we targeted galaxies below the Galactic latitude limit of 6dFGS and filled gaps in their coverage. 

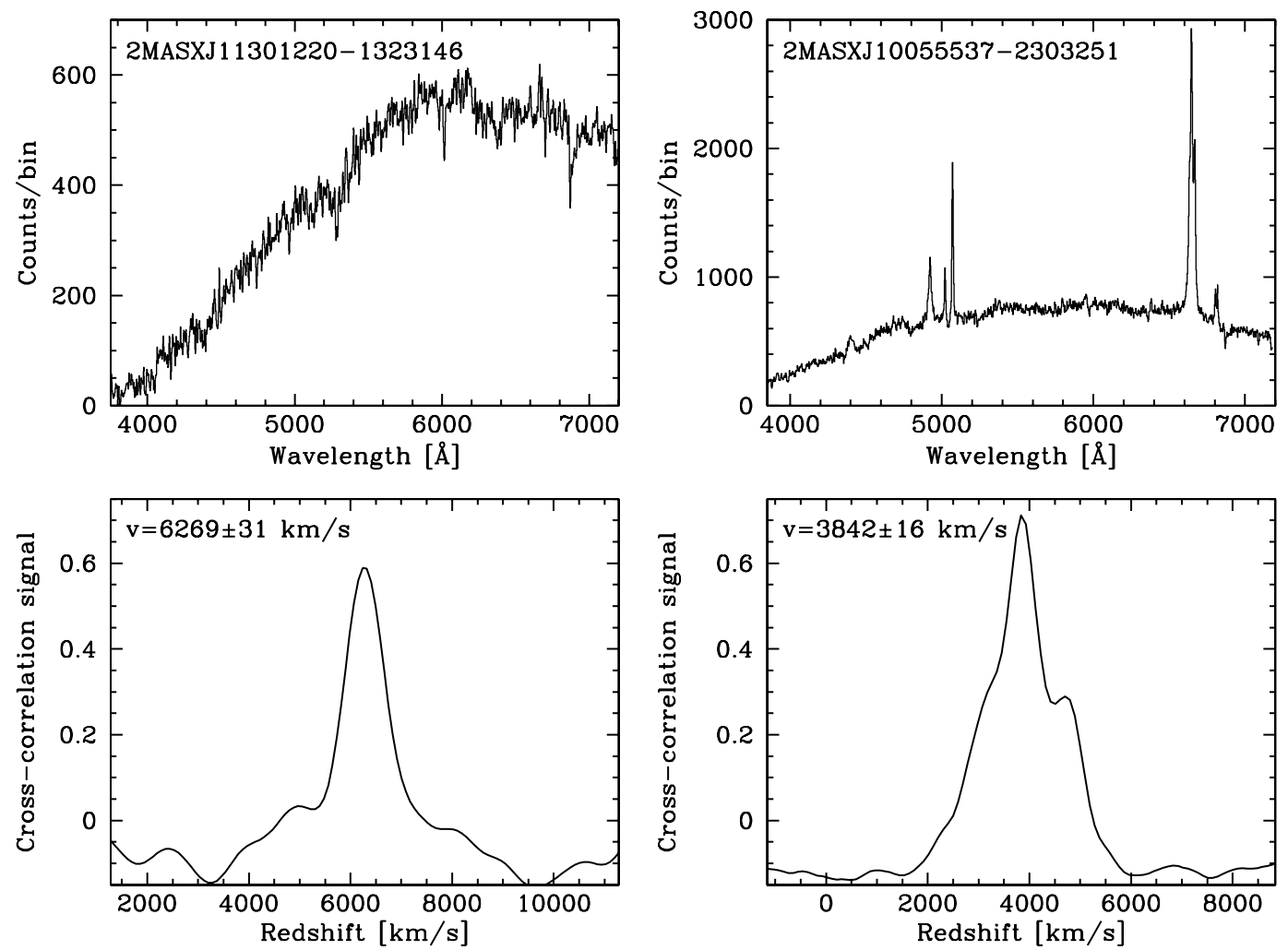

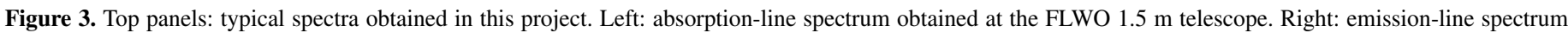
obtained at the CTIO $1.5 \mathrm{~m}$ telescope. Bottom panels: results of the cross-correlation technique used to measure the redshifts.

Table 2

Telescopes and Instruments Used in the Survey

\begin{tabular}{|c|c|c|c|c|c|c|c|}
\hline \multicolumn{2}{|c|}{ Observatory/Telescope } & \multirow[t]{2}{*}{ Camera } & \multirow{2}{*}{$\begin{array}{c}\text { Grating } \\
\left(1 \mathrm{~mm}^{-1}\right)\end{array}$} & \multirow{2}{*}{$\begin{array}{c}\text { Coverage } \\
\text { (§) }\end{array}$} & \multirow{2}{*}{$\begin{array}{l}\text { Res. } \\
(\AA)\end{array}$} & \multicolumn{2}{|c|}{ No. of Gals. with $K_{s}$} \\
\hline & & & & & & $<11.75$ & $>11.75$ \\
\hline Fred L. Whipple & $1.5 \mathrm{~m}$ & FAST & 300 & $3500-7400$ & 5 & 7590 & 2596 \\
\hline Cerro Tololo & $1.5 \mathrm{~m}$ & RCSpec & 300 & $3700-7200$ & 7 & 3245 & 238 \\
\hline McDonald & $2.1 \mathrm{~m}$ & es2 & 600 & $3700-6400$ & 4 & 114 & 50 \\
\hline Cerro Tololo & $4 \mathrm{~m}$ & RCSpec & 527 & $3700-7400$ & 3 & 48 & \\
\hline Hobby-Eberly & $9.2 \mathrm{~m}$ & LRS & 300 & $4300-10800$ & 9 & 3 & \\
\hline
\end{tabular}

At FLWO, most observations were carried out by P. Berlind and M. Calkins, with additional observations by J. Huchra, L. Macri, A. Crook, and E. Falco. Additional spectra were obtained in queue mode by other CfA-affiliated observers. At CTIO, observations were carried out by J. Huchra, L. Macri, and the SMARTS consortium queue operators. At McDonald, observations were carried out by J. Mader, T. George, and resident astronomers. Exposure times ranged from $120 \mathrm{~s}$ to $2400 \mathrm{~s}$ with an average value of $550 \mathrm{~s}$. Some galaxies were observed on multiple nights (sometimes with increased exposure times relative to the first exposure) to improve the quality of the redshift measurement. The total "open shutter" time for the observations was approximately $2100 \mathrm{hr}$. Bias and flat frames (dome or internal quartz lamp) were obtained daily. Comparison spectra were obtained before or after each science exposure using a variety of $\mathrm{He}, \mathrm{Ne}$, and Ar lamps. Stellar and galaxy radial velocity standards were observed nightly.

The spectra were reduced and analyzed in a uniform manner using IRAF. ${ }^{17}$ Images were debiased and flat-fielded using rou-

\footnotetext{
17 IRAF is distributed by the National Optical Astronomy Observatory, which is operated by the Association of Universities for Research in Astronomy (AURA) under cooperative agreement with the National Science Foundation.
}

tines in the CCDRED package and one-dimensional spectra were extracted using routines in the APEXTRACT package. Dispersion functions were derived from the comparison lamp spectra and applied to the observations using routines in the ONEDSPEC package. The spectra obtained at FLWO were processed by S. Tokarz and N. Martimbeau using the automated pipeline described in Tokarz \& Roll (1997). Two typical spectra are shown in the top panels of Figure 3.

Radial velocities were measured by the usual technique of cross-correlating spectra against templates (Tonry \& Davis 1979) using the XCSAO task in the RVSAO package (Kurtz \& Mink 1998). We used a variety of templates developed at the HarvardSmithsonian Center for Astrophysics. The bottom panels of Figure 3 show the results of the cross-correlation technique for the two representative spectra. Figure 4 shows histograms of internal velocity uncertainties for the galaxies observed at FLWO and CTIO. The median uncertainty values for spectra that only contain absorption lines are 29 and $41 \mathrm{~km} \mathrm{~s}^{-1}$ for FLWO and CTIO, respectively, while the corresponding values for emission-line spectra are 12 and $24 \mathrm{~km} \mathrm{~s}^{-1}$.

The reduced spectra are available for further analysis at the Smithsonian Astrophysical Observatory Telescope Data 


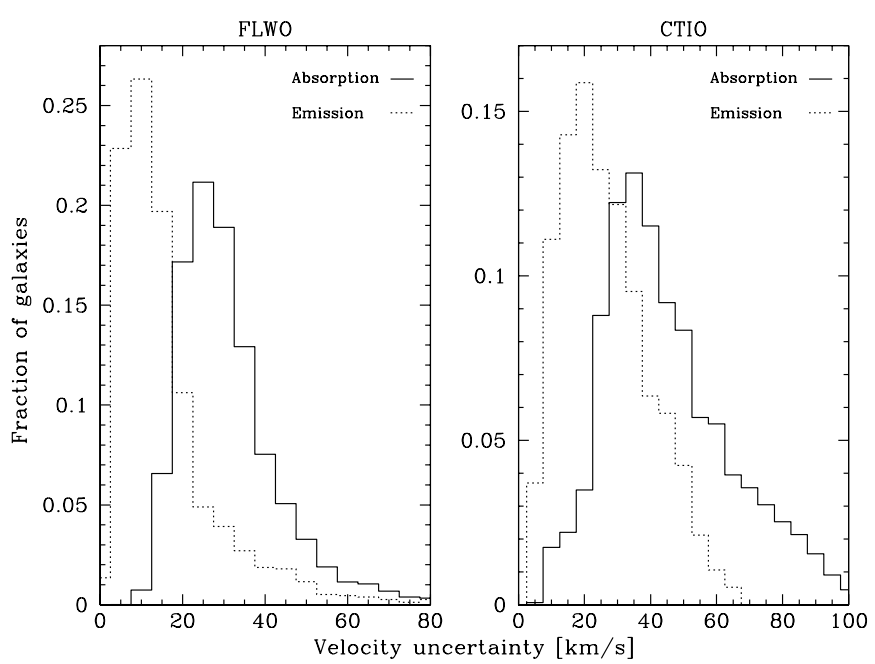

Figure 4. Distribution of velocity uncertainties for the galaxies observed at FLWO (left) and CTIO (right). The samples are further divided according to the absence or presence of emission lines.

Center $^{18}$ (hereafter, the 2 MRS Web site). For example, a list of galaxies with emission-line features is available for those interested in searching for nearby active galactic nucleus (AGN).

\subsection{Matching with Previous Redshift Catalogs}

We retrieved the SDSS-DR8 spectroscopic catalog ${ }^{19}$ and searched for counterparts to 2MASS sources using a tolerance radius of 2 .'5. We found 7069 matches to galaxies without 2MRS redshifts (including 390 galaxies with multiple SDSS observations for which we calculated a weighted mean redshift). These are identified with the catalog code "S."

We retrieved the 6dFGS-DR3 spectroscopic catalog ${ }^{20}$ and searched for counterparts to 2MASS sources using a tolerance radius of $10^{\prime \prime}$. We only selected redshifts measured with the $6 \mathrm{dF}$ instrument (code $=126$ in column 17 of their catalog), with velocity quality 3 or 4 (equivalent to velocity uncertainties of 55 and $45 \mathrm{~km} \mathrm{~s}^{-1}$, respectively). We obtained 11,763 matches to galaxies without $2 \mathrm{MRS}$ redshifts. These are identified with the catalog code " 6 ."

We performed a literature search for galaxies without 2MRS, $6 \mathrm{dF}$, or SDSS redshifts using the NASA Extragalactic Database (NED). First, we carried out a "Search by Name" query using the 2MASS IDs of the galaxies as input. This returned 12,694 redshifts that were incorporated into our catalog. We refer to these redshifts as the "NED default" set, and they are identified with the catalog code "N." Next, we performed a "Search near Position" query using the 2MASS coordinates of the galaxies for which no redshift information had been returned by the previous query. We used a tolerance radius of 1 '.3 for the search, which resulted in an additional 226 redshifts. These are galaxies where the difference in coordinates between 2MASS and previous catalogs is sufficiently large that NED has two or more entries for the same object, in most cases "associated" (in NED terms) with one another but no redshift information is returned when querying by 2 MASS ID. In the case of an additional 32 galaxies, we did not use the default redshift returned by NED but

\footnotetext{
18 http://tdc-www.cfa.harvard.edu/2mrs/

19 http://data.sdss3.org/sas/dr8/common/sdss-spectro/ redux/galSpecInfo-dr8.fits

20 http://www-wfau.roe.ac.uk/6dFGS/6dFGSzDR3.txt.gz
}

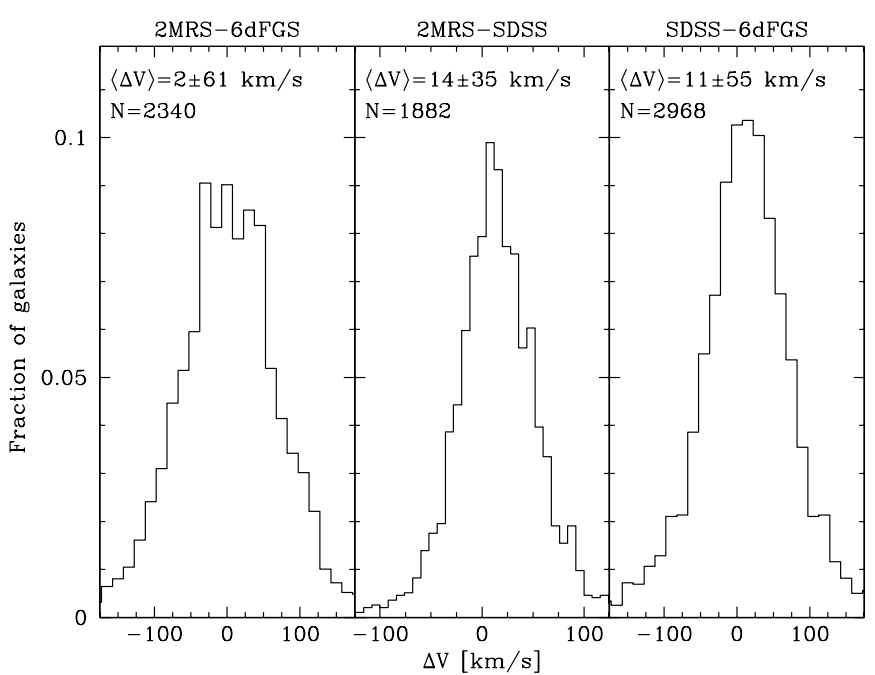

Figure 5. Histogram of velocity differences for galaxies observed by any two of $2 \mathrm{MRS}, 6 \mathrm{dFGS}$, or SDSS.

instead adopted an alternative redshift listed in NED. These 258 "alternative NED redshifts" are identified with the catalog code "M."

Lastly, we matched the 2MASS XSC against J. Huchra's personal compilation of redshifts (ZCAT) and found velocities for an additional 749 galaxies which had no corresponding information in NED, including 455 galaxies observed by J. Huchra or collaborators prior to the start of 2MRS but were never published. We also identified 77 galaxies for which the ZCAT and NED redshifts were in disagreement and we gave preference to the ZCAT values. Detailed information on these galaxies and those for which we assigned alternative NED redshifts (see preceding paragraph) is provided in the Appendix. Galaxies with ZCAT redshifts are identified with catalog code "O."

Our catalog gives preference to $2 \mathrm{MRS}$ redshifts over any previously published SDSS or $6 \mathrm{dF}$ value, to SDSS over $6 \mathrm{dF}$, to $6 \mathrm{dF}$ over NED, and to NED over ZCAT, except for the cases described above. We list the additional redshifts for galaxies with multiple measurements in the Appendix, to allow interested readers to assign a different set of precedences or to compute weighted mean redshifts.

Figure 5 shows a comparison of redshifts for all 2MASS galaxies observed by us and by 6dFGS or SDSS. The average redshift difference for galaxies in common between each pair of catalogs is the following: $2 \pm 61 \mathrm{~km} \mathrm{~s}^{-1}$ for $N=2511$ galaxies in 2MRS and 6dFGS; $14 \pm 35 \mathrm{~km} \mathrm{~s}^{-1}$ for $N=1940$ galaxies in 2MRS and SDSS; $11 \pm 55 \mathrm{~km} \mathrm{~s}^{-1}$ for $N=3187$ galaxies in 6dFGS and SDSS. The dispersions are consistent with the typical velocity uncertainties of each survey $\left(30-40 \mathrm{~km} \mathrm{~s}^{-1}\right.$ for 2MRS, 45-55 $\mathrm{km} \mathrm{s}^{-1}$ for $6 \mathrm{dFGS}$, and $5 \mathrm{~km} \mathrm{~s}^{-1}$ for SDSS).

\subsection{The 2MRS Catalog}

The 2MRS catalog is presented in Table 3 and is also available at the 2MRS Web site. It contains 29 columns that are described below, including the original 2MASS XSC column names in square brackets when applicable.

1. ID: 2MASS ID [designation]

2. R.A.: right ascension (deg, J2000.0) [sup_ra]

3. Decl.: declination (deg, J2000.0) [sup_dec]

4. $l$ : Galactic longitude 
Table 3

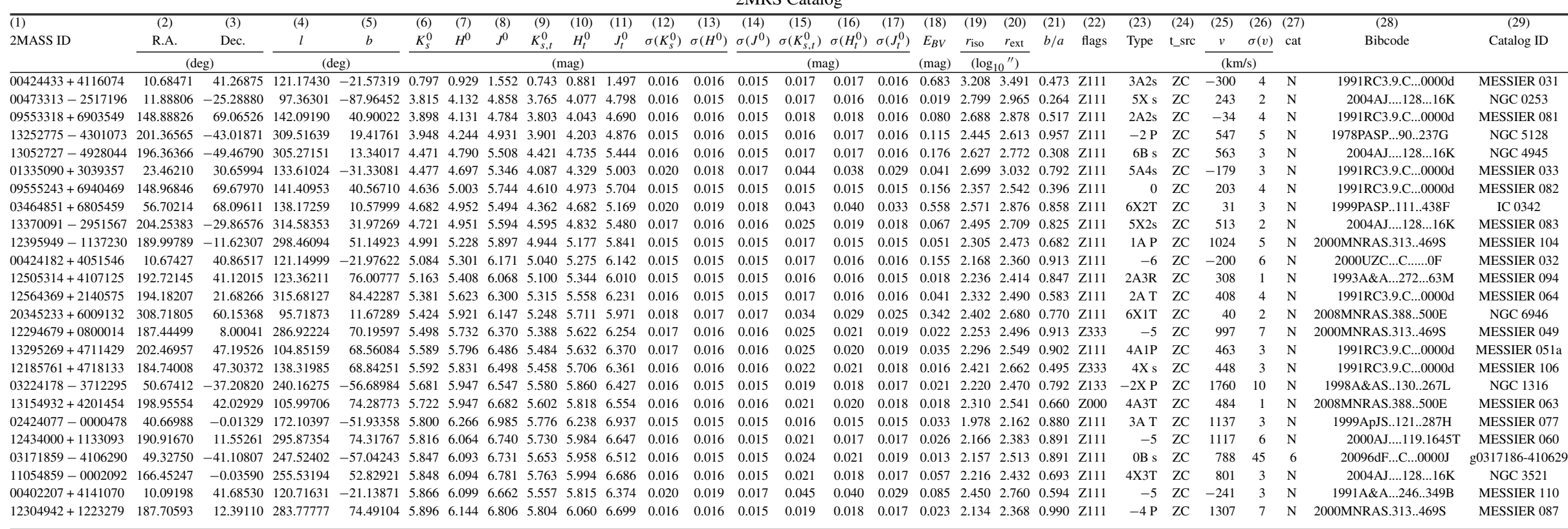

Notes. Codes for Column 27: [C]TIO, Mc[D]onald, [F]LWO, [N]ED 2MASS ID match, NED position [M]atch, [O]ther sources in ZCAT, [S]DSS-DR8, and [6]dFGS.

(This table is available in its entirety in a machine-readable form in the online journal. A portion is shown here for guidance regarding its form and content.) 
Table 4

2MRS Catalog_Bibliographic References

\begin{tabular}{ll}
\hline \hline ADS Bibcode & \multicolumn{1}{c}{ Reference } \\
\hline 1969MSAIt..40..559B & Barbon (1969) \\
1970ApJ...160..405S & Sargent (1970) \\
1970ApJ...160L..33B & Burbidge (1970) \\
1971ApJ..168..321C & Chincarini \& Rood (1971) \\
1971CGPG..C...0000Z & Zwicky \& Zwicky (1971) \\
1972ApJ...172L..37B & Burbidge \& Strittmatter (1972) \\
1972ApJ...173..247S & Stockton (1972) \\
1972AuJPh..25..233W & Whiteoak (1972) \\
1972IAUS...44..376L & Lynds (1972) \\
1972MNRAS.158..277T & Tritton (1972) \\
\hline
\end{tabular}

(This table is available in its entirety in a machine-readable form in the online journal. A portion is shown here for guidance regarding its form and content.)

5. $b$ : Galactic latitude

6. $K_{s}^{0}$ : extinction-corrected $K_{s}$ isophotal magnitude [k_m_ $\mathrm{k} 20 \mathrm{fe}]$

7. $H^{0}$ : same for $\mathrm{H}$ [h_m_k20fe]

8. $J^{0}$ : same for J [j_m_k20fe]

9. $K_{s, t}^{0}:$ extinction-corrected "total" extrapolated $K_{s}$ magnitude [k_m_ext]

10. $H_{t}^{0}:$ same for $\mathrm{H}$ [h_m_ext]

11. $J_{t}^{0}$ : same for $\mathrm{J}\left[\mathrm{j} \_\mathrm{m} \_\right.$ext $]$

12. $\sigma\left(K_{s}^{0}\right)$ : uncertainty in $K_{s}^{0}$ [k_msig_k20fe]

13. $\sigma\left(H^{0}\right)$ : same for $H^{0}$ [h_msig_k20fe]

14. $\sigma\left(J^{0}\right)$ : same for $J^{0}$ [j_msig_k20fe]

15. $\sigma\left(K_{s, t}^{0}\right)$ : same for $K_{t}^{0}\left[\mathrm{k} \_\mathrm{msig} \_\mathrm{ext}\right]$

16. $\sigma\left(H_{t}^{0}\right)$ : same for $H_{t}^{0}$ [h_msig_ext]

17. $\sigma\left(J_{t}^{0}\right)$ : same for $J_{t}^{0}$ [j_msig_ext]

18. $E(B-V)$ : from Schlegel et al. (1998)

19. $r_{\text {iso }}: \log _{10}$ of the $K_{s}=20 \mathrm{mag} / \mathrm{sq}$. arcsec isophotal radius (in arcseconds) [r_k20fe]

20. $r_{\text {ext }}$ : same as $r_{\text {iso }}$ but for "total magnitude" extrapolation radius [r_ext]

21. $b / a$ : axial ratio from co-added $J H K_{s}$ images [sup_ba]

22. flags: photometry confusion flags from 2MASS XSC database. "Z" in the first column indicates magnitudes from the 2MASS LGA. [cc_flg, k_flg_k20fe, h_flg_k20fe, j_flg_k20fe].

23. type: galaxy type (see Section 5 and Table 5)

24. t_src: source of galaxy type $(\mathrm{JH}=\mathrm{John}$ Huchra; $\mathrm{ZC}=$ ZCAT; $\mathrm{NN}=$ not available)

25. v: redshift $\left(\mathrm{km} \mathrm{s}^{-1}\right.$, barycentric)

26. $\sigma(v)$ : uncertainty in redshift $\left(\mathrm{km} \mathrm{s}^{-1}\right)$

27. cat: code for redshift catalog (see notes for details).

28. v_src: NED bibliographic code for source of redshift (see Table 4 for references)

29. Catalog ID: galaxy ID in redshift catalog

In addition to our measurements, Table 3 contains redshifts from 578 publications which are referenced in the catalog using ADS/NED bibliographic codes (see Table 4). We strongly encourage proper citation of the original publications when making use of any of these values.

Table 6 lists 4291 redshifts for 2MASS galaxies which lie beyond the limits of our main catalog; 2884 were observed as part of this project while 1407 had been previously targeted by J. Huchra and collaborators for other projects. Lastly, Table 7 presents redshifts for 14 galaxies that are not in the 2MASS XSC but which were observed serendipitously due to their proximity to our targets.
Table 5

Morphological Type Codes used in 2MRS

\begin{tabular}{|c|c|}
\hline Code & Comment \\
\hline \multicolumn{2}{|r|}{ Types } \\
\hline-9 & QSO/AGN \\
\hline-7 & Unclassified Elliptical \\
\hline-6 & Compact Elliptical \\
\hline-5 & $\mathrm{E}$, and dwarf $\mathrm{E}$ \\
\hline-4 & $\mathrm{E} / \mathrm{SO}$ \\
\hline-3 & L-, SO- \\
\hline-2 & $\mathrm{~L}, \mathrm{SO}$ \\
\hline-1 & $\mathrm{~L}+, \mathrm{SO}+$ \\
\hline 0 & $\mathrm{SO} / \mathrm{a}, \mathrm{SO}-\mathrm{a}$ \\
\hline 1 & $\mathrm{Sa}$ \\
\hline 2 & $\mathrm{Sab}$ \\
\hline 3 & $\mathrm{Sb}$ \\
\hline 4 & Sbc \\
\hline 5 & $\mathrm{Sc}$ \\
\hline 6 & Scd \\
\hline 7 & $\mathrm{Sd}$ \\
\hline 8 & $\mathrm{Sdm}$ \\
\hline 9 & $\mathrm{Sm}$ \\
\hline 10 & Im, Irr I, Magellanic Irregular, Dwarf Irregular \\
\hline 11 & Compact Irregular, Extragalactic H II Region \\
\hline 12 & Extragalactic H I cloud (no galaxy visible) \\
\hline 15 & Peculiar, Unclassifiable \\
\hline 16 & Irr II \\
\hline 19 & Unclassified galaxy (visually confirmed to be a galaxy, but not typed) \\
\hline 20 & S..., Sc-Irr, Unclassified Spiral \\
\hline 98 & Galaxy that has never been visually examined. \\
\hline \multicolumn{2}{|r|}{ Bar types } \\
\hline A & unbarred (A) \\
\hline $\mathrm{X}$ & mixed type (AB) \\
\hline B & barred (B) \\
\hline \multicolumn{2}{|r|}{ Peculiarities } \\
\hline $\mathrm{D}$ & Double or Multiple \\
\hline $\mathrm{P}$ & Peculiar \\
\hline $\mathrm{R}$ & Outer Ring \\
\hline $\mathrm{r}$ & Inner Ring \\
\hline $\mathrm{s}$ & S-shaped \\
\hline $\mathrm{t}$ & Mixed (Inner ring/S-shaped) \\
\hline $\mathrm{T}$ & Pseudo outer ring \\
\hline / & Spindle \\
\hline \multicolumn{2}{|r|}{ Luminosity classes (for spirals \& irregulars) } \\
\hline 1 & I \\
\hline 2 & I-II \\
\hline 3 & II \\
\hline 4 & II-II \\
\hline 5 & III \\
\hline 6 & III-IV \\
\hline 7 & IV \\
\hline 8 & IV-V \\
\hline 9 & $\mathrm{~V}$ \\
\hline
\end{tabular}

Notes. The morphological information is encoded in Table 3 following the ZCAT convention. It is a five digit code (I2, A1, I1, and A1). The first two digits are the numerically coded $\mathrm{T}$ type, the next letter (if present) is the Bar type, the next digit (if present) is the numerically coded luminosity class, and the final letter (if present) denotes morphological perculiarities.

Figure 6 shows the distribution of galaxies as a function of redshift for the 2MRS main sample and selected surveys from Table 1. 
Table 6

Redshifts for Galaxies in the 2MASS XSC Beyond the Main 2MRS Catalog Limits

\begin{tabular}{|c|c|c|c|c|c|c|}
\hline \multirow[t]{2}{*}{ 2MASS ID } & R.A. & Decl. & $v$ & $\sigma(v)$ & \multirow{2}{*}{$\begin{array}{l}\text { Vel } \\
\text { src. }\end{array}$} & \multirow{2}{*}{$\begin{array}{c}\text { Bibliographic } \\
\text { Code }\end{array}$} \\
\hline & \multicolumn{2}{|c|}{ (deg) } & \multicolumn{2}{|c|}{$\left(\mathrm{km} \mathrm{s}^{-1}\right)$} & & \\
\hline $00000256+0817537$ & 0.01063 & 8.29817 & 11721 & 37 & $\mathrm{O}$ & 20112MRS.MMT..0000H \\
\hline $00000896+0817338$ & 0.03729 & 8.29272 & 12280 & 36 & $\mathrm{O}$ & 20112MRS.MMT..0000H \\
\hline $00001215+0205503$ & 0.05069 & 2.09740 & 6506 & 35 & $\mathrm{O}$ & 20112MRS.JPH..0000H \\
\hline $00005299+0803392$ & 0.22079 & 8.06090 & 11917 & 35 & $\mathrm{O}$ & 20112MRS.JPH..0000H \\
\hline $00005467+0803442$ & 0.22779 & 8.06231 & 11952 & 10 & $\mathrm{O}$ & 20112MRS.JPH..0000H \\
\hline $00015848+1203580$ & 0.49370 & 12.06618 & 60938 & 55 & $\mathrm{O}$ & 20112MRS.JPH..0000H \\
\hline $00021610-2926230$ & 0.56700 & -29.43970 & 18213 & 40 & $\mathrm{C}$ & 20112MRS.CTIO.0000H \\
\hline $00023474-2948240$ & 0.64483 & -29.80667 & 17766 & 31 & $\mathrm{C}$ & 20112MRS.CTIO.0000H \\
\hline $00032067-3008493$ & 0.83607 & -30.14699 & 20407 & 38 & $\mathrm{C}$ & 20112MRS.CTIO.0000H \\
\hline $00033524+1700158$ & 0.89692 & 17.00435 & 16512 & 42 & $\mathrm{O}$ & 20112MRS.JPH..0000H \\
\hline $00033886+1129529$ & 0.91189 & 11.49804 & 17339 & 27 & $\mathrm{O}$ & 20112MRS.JPH..0000H \\
\hline $00042025+3120313$ & 1.08443 & 31.34198 & 5073 & 31 & $\mathrm{O}$ & 20112MRS.JPH..0000H \\
\hline $00052335-0810122$ & 1.34734 & -8.17015 & 9099 & 46 & $\mathrm{O}$ & 20112MRS.MMT..0000H \\
\hline
\end{tabular}

Note. Codes for Column 6: [C]TIO, Mc[D]onald, and [F]LWO.

(This table is available in its entirety in a machine-readable form in the online journal. A portion is shown here for guidance regarding its form and content.)

Table 7

Redshifts for Galaxies not in the 2MASS XSC which were Observed Serendipitously

\begin{tabular}{|c|c|c|c|c|c|c|}
\hline \multirow[t]{2}{*}{ 2MASS ID } & R.A. & Decl. & $v$ & $\sigma(v)$ & \multirow{2}{*}{$\begin{array}{l}\text { Vel } \\
\text { src. }\end{array}$} & \multirow{2}{*}{$\begin{array}{l}\text { Bibliographic } \\
\text { Code }\end{array}$} \\
\hline & \multicolumn{2}{|c|}{$(\operatorname{deg})$} & \multicolumn{2}{|c|}{$\left(\mathrm{km} \mathrm{s}^{-1}\right)$} & & \\
\hline $13294821-2340551$ & 202.45088 & -23.68197 & 5029 & 52 & $\mathrm{C}$ & 20112MRS.CTIO.0000H \\
\hline $10245589-1726331$ & 156.23289 & -17.44251 & 7811 & 43 & $\mathrm{C}$ & 20112MRS.CTIO.0000H \\
\hline $10104329-1530289$ & 152.68038 & -15.50803 & 8388 & 29 & $\mathrm{C}$ & 20112MRS.CTIO.0000H \\
\hline $01574403-0649389$ & 29.43346 & -6.82746 & 8434 & 21 & $\mathrm{C}$ & 20112MRS.CTIO.0000H \\
\hline $10324638-1238045$ & 158.19324 & -12.63459 & 8491 & 45 & $\mathrm{C}$ & 20112MRS.CTIO.0000H \\
\hline $10324555-1238140$ & 158.18977 & -12.63721 & 8579 & 43 & $\mathrm{C}$ & 20112MRS.CTIO.0000H \\
\hline $11111075-0628055$ & 167.79479 & -6.46819 & 8676 & 14 & $\mathrm{C}$ & 20112MRS.CTIO.0000H \\
\hline $03452702-0728392$ & 56.36260 & -7.47756 & 10504 & 31 & $\mathrm{C}$ & 20112MRS.CTIO.0000H \\
\hline $16265144-7709323$ & 246.71447 & -77.15896 & 13333 & 41 & $\mathrm{C}$ & 20112MRS.CTIO.0000H \\
\hline $16311208-2615525$ & 247.80033 & -26.26458 & 13380 & 55 & $\mathrm{C}$ & 20112MRS.CTIO.0000H \\
\hline $03190550-2140362$ & 49.77291 & -21.67673 & 15624 & 32 & $\mathrm{C}$ & 20112MRS.CTIO.0000H \\
\hline $01135502-3659473$ & 18.47924 & -36.99647 & 15814 & 56 & $\mathrm{C}$ & 20112MRS.CTIO.0000H \\
\hline $16385916-6421059$ & 249.74644 & -64.35169 & 16320 & 46 & $\mathrm{C}$ & 20112MRS.CTIO.0000H \\
\hline $23342933-6921154$ & 353.62221 & -69.35433 & 25413 & 49 & $\mathrm{C}$ & 20112MRS.CTIO.0000H \\
\hline
\end{tabular}

Note. ${ }^{a}$ Pseudo-2MASS ID generated from the celestial coordinates of the object.

(This table is available in its entirety in a machine-readable form in the online journal. A portion is shown here for guidance regarding its form and content.)

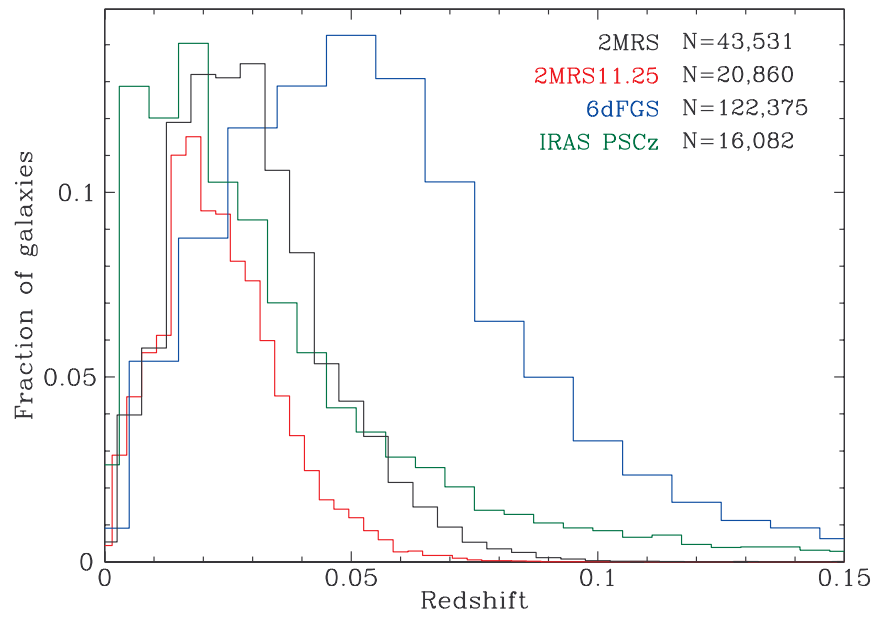

Figure 6. Distribution of galaxies as a function of redshift for $2 \mathrm{MRS}$ and for some of the redshift surveys listed in Table 1.

(A color version of this figure is available in the online journal.)

\section{COSMIC CARTOGRAPHY}

Some initial qualitative results from this survey are shown below via two visualization techniques: Hockey Pucks and Onion Skins.

\subsection{Hockey Pucks}

An all-sky survey allows us to make plots of the nearby galaxy distribution that are more representative than simple strip surveys (de Lapparent et al. 1986). The angular nature of strips around the sky, when projected onto a plane, are somewhat deceptive of real structure. They are thin at the center and thick at the edge. While this partially makes up for the normal decrease in the selection efficiency as a function of redshift in a flux-limited sample, it provides a representation of structure that varies quite strongly from the center to edge. With full-sky coverage, it is possible to project actual cylinders of redshift space. Given the long-term association of redshift surveys with the Harvard-Smithsonian CfA, we naturally call these "Hockey Puck" plots. Code to generate these plots is available as part of the 2MRS data release. 


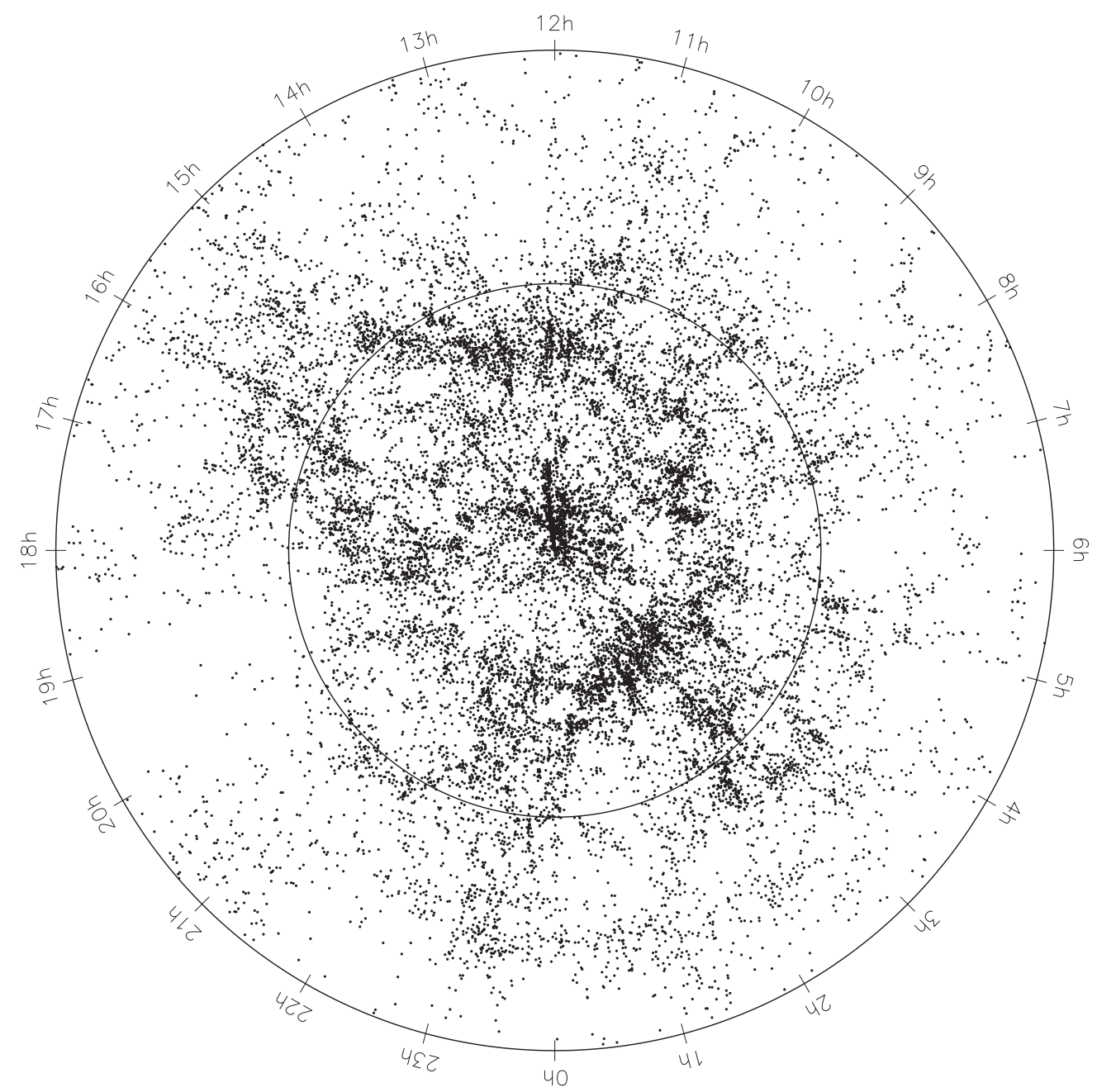

Figure 7. Hockey Puck plot—a full cylinder section—of 2MRS in the north celestial cap. The view is looking downward from the NCP, the thickness of the "puck" is $8000 \mathrm{~km} \mathrm{~s}^{-1}$, and its radius is $15,000 \mathrm{~km} \mathrm{~s}^{-1}$.

Two "Hockey Puck" diagrams shown in Figures 7 and 8 highlight the vast improvement in coverage through the galactic plane afforded by $2 \mathrm{MRS}$ as compared to even CfA2, the densest survey of the nearby universe (Huchra et al. 1995, 1999). Plotted are top-down views of cylindrical volumes with a radius of $15,000 \mathrm{~km} \mathrm{~s}^{-1}$ and thickness of $8000 \mathrm{~km} \mathrm{~s}^{-1}$, yielding an aspect ratio of about 3.5-1. The pucks show the galaxies in the northern and southern celestial hemispheres, respectively-i.e., all galaxies above and below the celestial equator with redshifts placing them in the cylinder and with $K_{s} \leqslant 11.75$ mag. Many of our favorite structures and several prominent voids are easily seen in these plots.

The northern puck is dominated by the LSC at the center, the Great Wall (now straight in this cylindrical projection) at 10-14.5 hr, and Pisces-Perseus at 0-5 hr. In addition, there are several new but smaller structures such as the one at $19 \mathrm{hr}$ and $4000 \mathrm{~km} \mathrm{~s}^{-1}$, probably best associated with the Cygnus Cluster (Huchra et al. 1977).

The southern celestial hemisphere is more amorphous. There is the well-known Cetus Wall (Fairall et al. 1998) between 0 and $4 \mathrm{hr}$, the southern part of the LSC at the center, and the Hydra-Centaurus region, but also a large and diffuse overdensity between 19 and $22 \mathrm{hr}$, a region hitherto not mapped because of its proximity to the galactic plane. This structure appears to be both large and rich and should have a large effect on the local velocity field.

\subsection{Onion Skins}

Another projection that can highlight the properties of nearby structures are surface maps of the galaxy distribution as a function of redshift. Since these are conceptually like peeling an onion, they are best called "Onion Skins." Figures 9-11 show three sets of these skins, moving progressively outward in redshift, while Figure 12 shows the entire 2MRS catalog with the major structures of the Local Universe labeled. These figures use Galactic coordinate projections; the corresponding equatorial coordinate projections are shown in Figures 15-18.

Figure 9 shows the distribution on the sky of all galaxies in the survey inside $3000 \mathrm{~km} \mathrm{~s}^{-1}$ color coded by redshift in $1000 \mathrm{~km} \mathrm{~s}^{-1}$ skins. The plane of the LSC dominates the map, but there is also a diffuse component between 2000 and $3000 \mathrm{~km} \mathrm{~s}^{-1}$ and $6-13 \mathrm{hr}$ in the south. The next two figures again show some familiar structures but with a few surprises. The Great Wall, Pisces-Perseus, and the Great Attractor dominate the mid ranges. The overdensity of galaxies in the direction of A3627 is high, and the comparison of Figure 10 with 11 clearly shows why we are moving with respect to the CMB toward a point around $l=270^{\circ}$ and $b=30^{\circ}$.

\section{GALAXY MORPHOLOGIES}

Morphological types are listed in Table 3 for all of the 20,860 galaxies in 2MRS11.25. We used the classifications listed in 


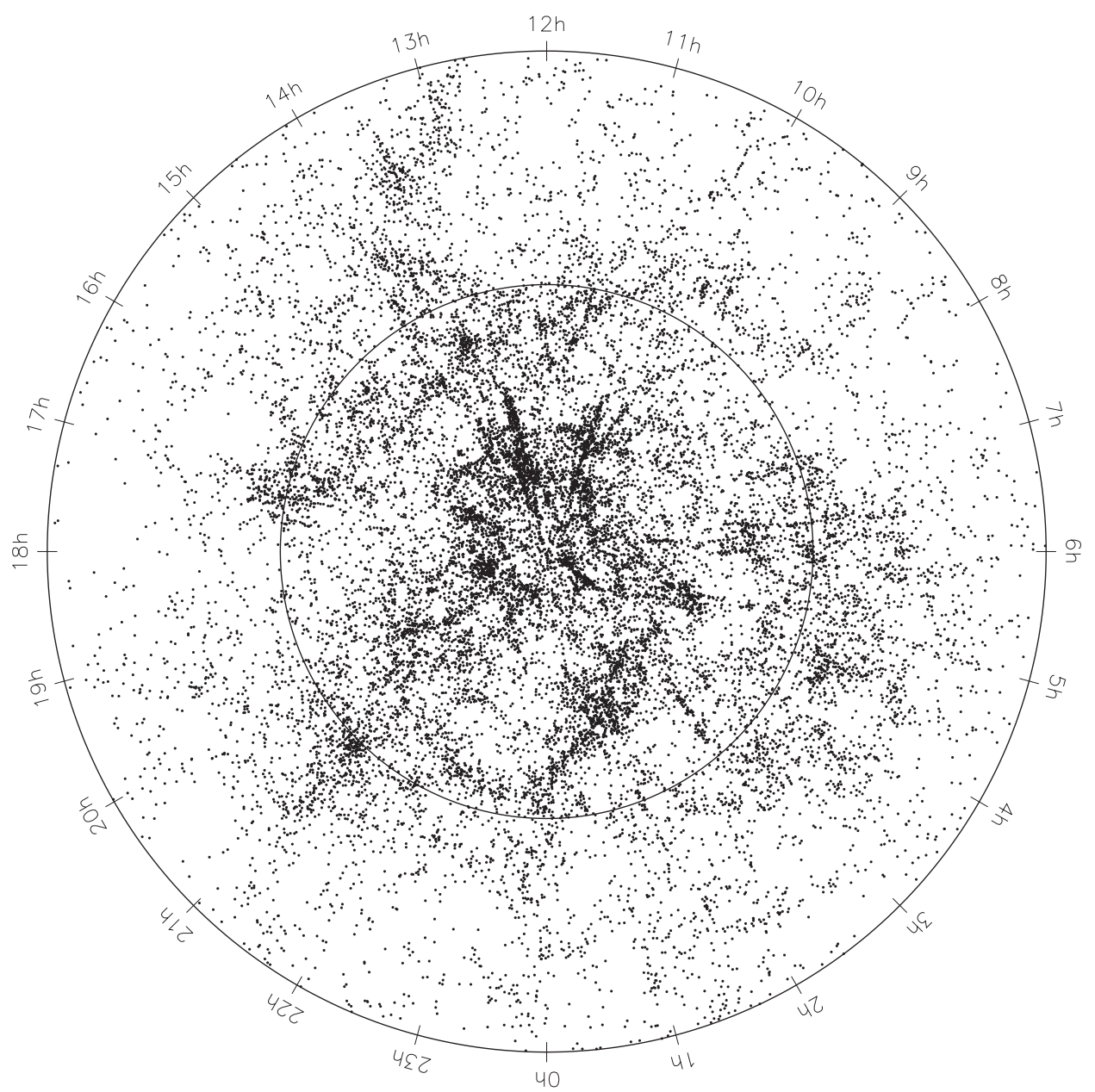

Figure 8. Same as Figure 7 but for the south celestial cap.

ZCAT (based on RC3, NED, and other catalogs) when available, but 5682 of these galaxies had no type information. They were visually examined and classified by J. Huchra using blue plates from the Digitized Sky Surveys. These new morphological types are identified by code "JH" in column 24 of the catalog. We also list morphological types from the literature for fainter galaxies in the catalog, when available.

Morphological typing in 2MRS uses the modified Hubble sequence (de Vaucouleurs 1963; de Vaucouleurs et al. 1976). Elliptical galaxies have integer types -7 through -5 . $\mathrm{S} 0$ galaxies range from integer type $-4(\mathrm{E} / \mathrm{S} 0)$ through 0 (SO/ a), in a sequence from least to most pronounced disks. Spirals are assigned integer types $1(\mathrm{Sa})$ through $9(\mathrm{Sm})$, without distinction between barred, unbarred or mixed-type. Irregular and peculiar galaxies are assigned integer types 10 and above. The format for the morphological type designations is described in detail in Table 5.

The distribution of the galaxies in 2MRS11.25 by morphological type is shown in Figure 13, while Figure 14 shows histograms by redshift for the three broad morphological classes described above. While the histograms show the same pattern as Figure 6, spirals dominate the data set at lower redshifts, while ellipticals flatten near $z \approx 0.03$ and extend to higher redshifts, as expected given their higher luminosity.

\section{PREVIOUS RESULTS FROM 2MRS}

The 2MRS11.25 sample has been used in several publications.
1. Erdoğdu et al. (2006a) calculated the acceleration on the Local Group (LG). Their estimate of the dipole seems to converge to the $\mathrm{CMB}$ result within $60 \mathrm{~h}^{-1} \mathrm{Mpc}$, suggesting that the bulk of the motion of the LG comes from structures within that distance. They also carried out an analysis of the dipole weighting the sample by its luminosity (rather than the counts) and found relatively minor changes.

2. Erdoğdu et al. (2006b) calculated density and velocity fields. All major LSCs and voids were successfully identified, and backside infall on to the "Great Attractor" region (at $50 h^{-1} \mathrm{Mpc}$ ) was detected.

3. Westover (2007) measured the correlation function and found a steeper relationship between galaxy bias and luminosity than previously determined for optical samples, implying that near-infrared luminosities may be better mass tracers than optical ones. The relative biasing between early- and late-type galaxies was best fit by a power law with no improvement when stochasticity was added, leaving open the possibility that populations of galaxies may evolve between one another.

4. Crook et al. (2007) produced a catalog of galaxy groups, which was later used to model the local velocity field in Crook et al. (2010).

5. Erdoğdu \& Lahav (2009) predicted the acceleration of the LG generated by $2 \mathrm{MRS}$ in the framework of $\Lambda \mathrm{CDM}$ and the halo model of galaxies. Their analysis suggested that it is not necessary to invoke additional unknown mass 


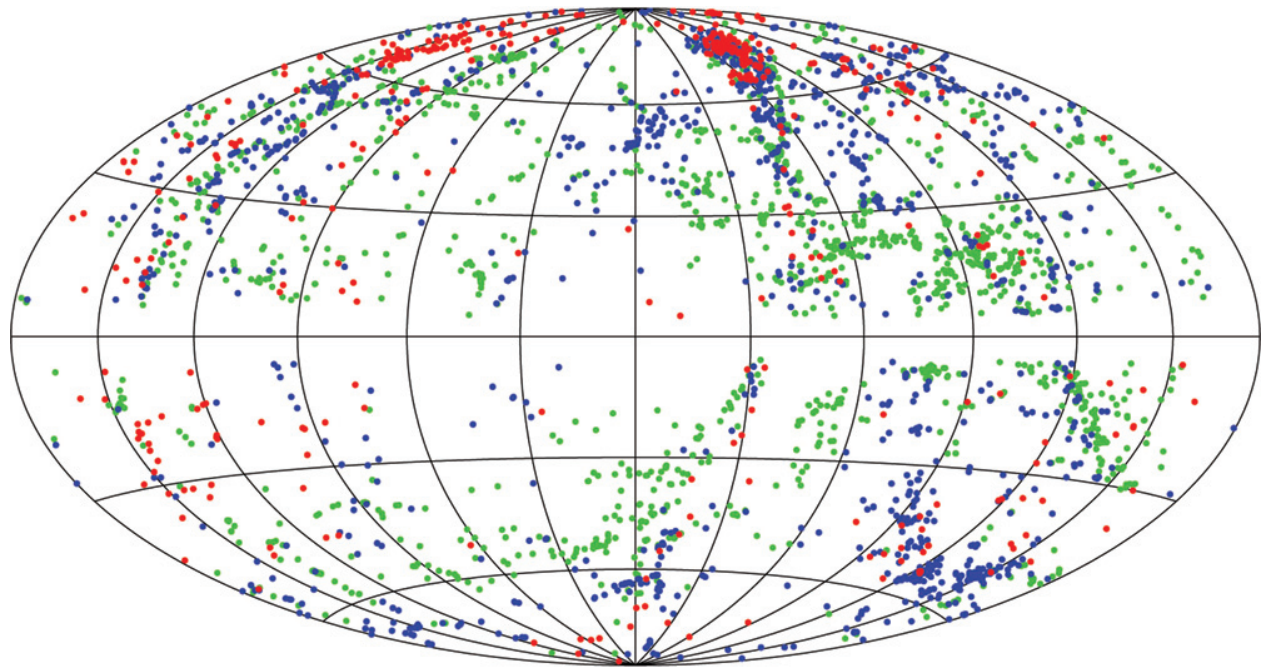

Figure 9. 2MASS galaxies inside the $3000 \mathrm{~km} \mathrm{~s}^{-1}$ sphere in Galactic coordinates (centered at $l=0^{\circ}$ and following the convention of $l$ increasing to the left). Heliocentric velocities are color coded with red, blue, and green representing bins of increasing redshift/distance. Red for $V_{h}<1000 \mathrm{~km} \mathrm{~s}^{-1}$, blue for $1000<V_{h}<2000 \mathrm{~km} \mathrm{~s}{ }^{-1}$, and green for $2000<V_{h}<3000 \mathrm{~km} \mathrm{~s}^{-1}$.

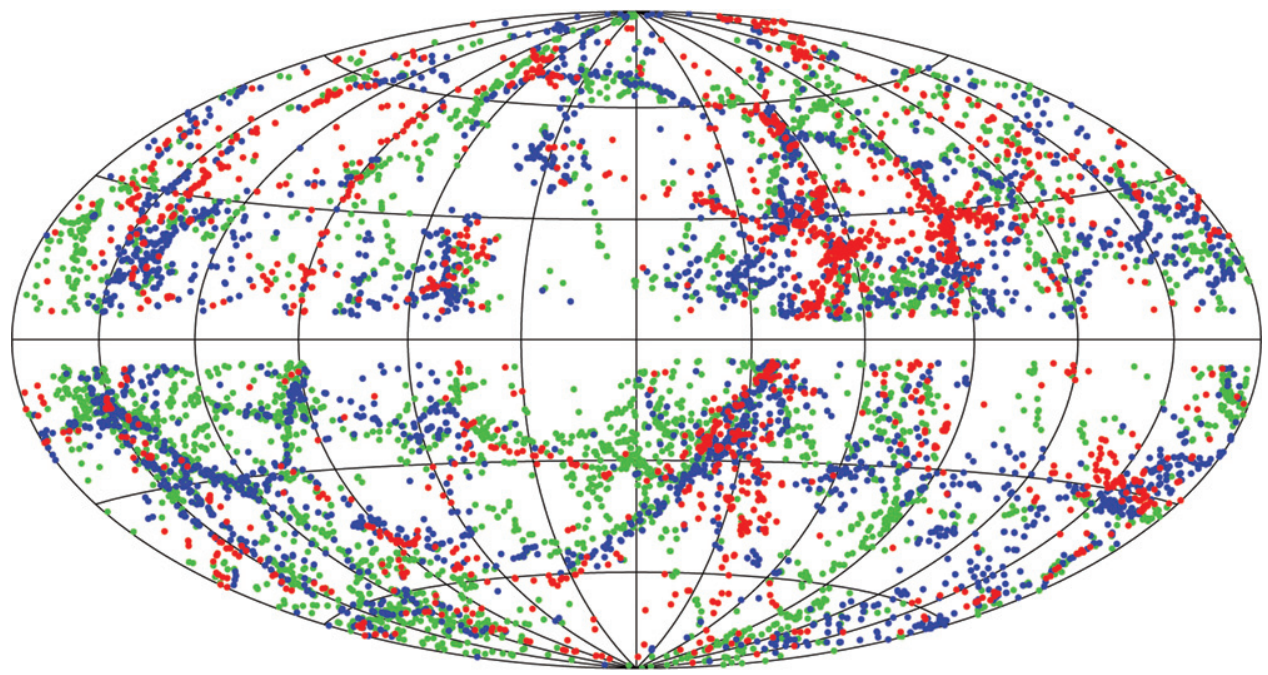

Figure 10. Same as Figure 9, but for velocities between 3000 and $6000 \mathrm{~km} \mathrm{~s}^{-1}$.

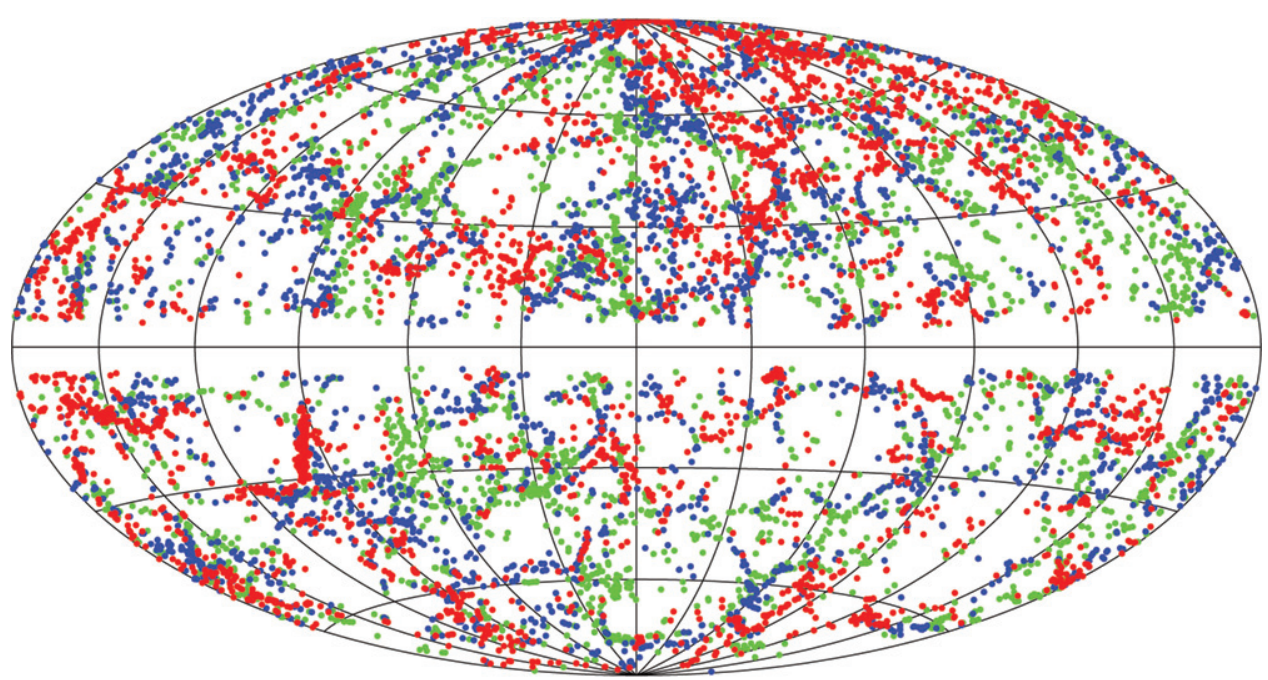

Figure 11. Same as Figure 9, but for velocities between 6000 and $9000 \mathrm{~km} \mathrm{~s}^{-1}$. 


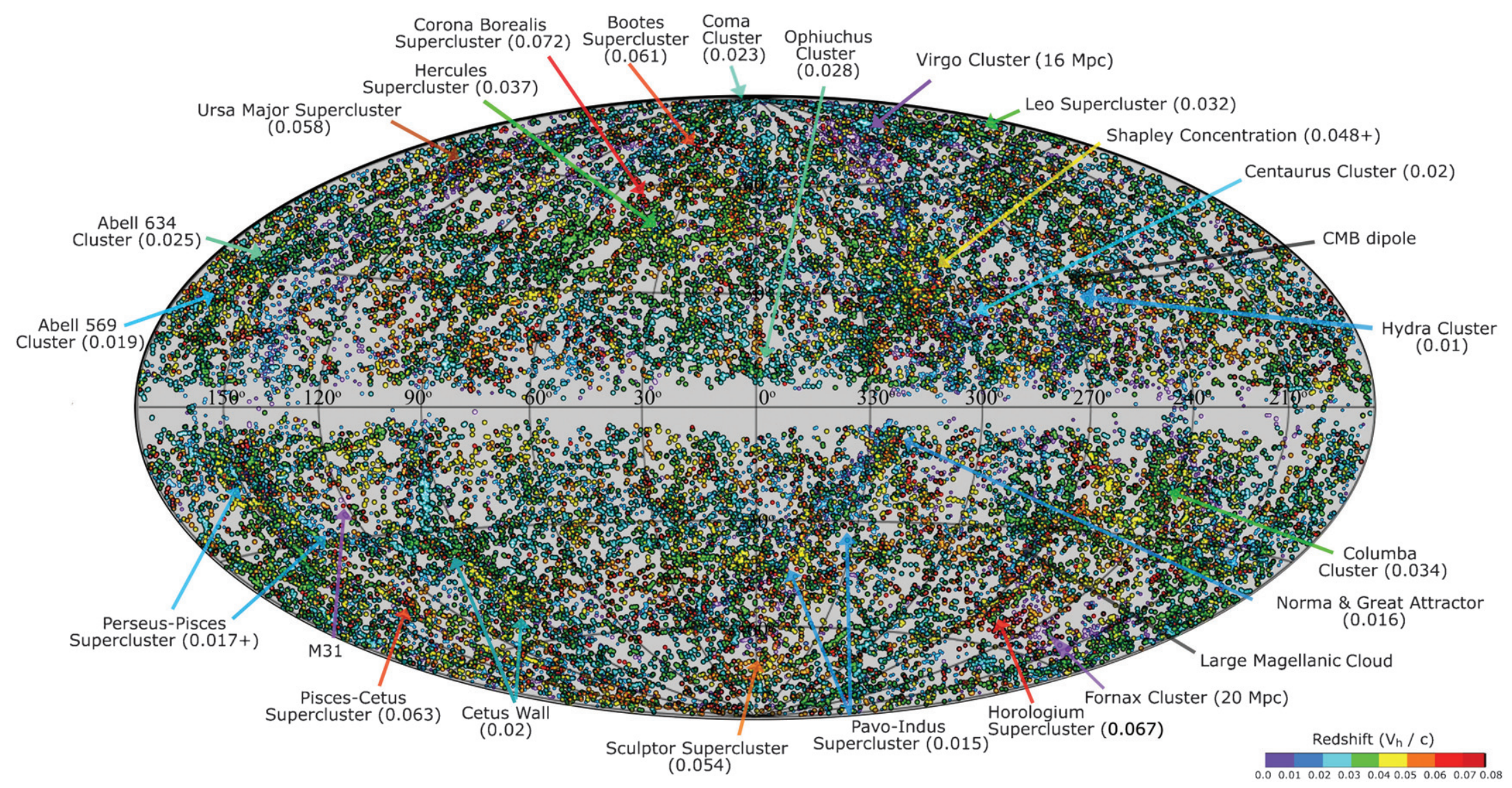

Figure 12. Same as Figure 9 but for all 2MRS galaxies, spanning the entire redshift range covered by the survey (from $z=0$ in purple to $z=0.08$ in red). 


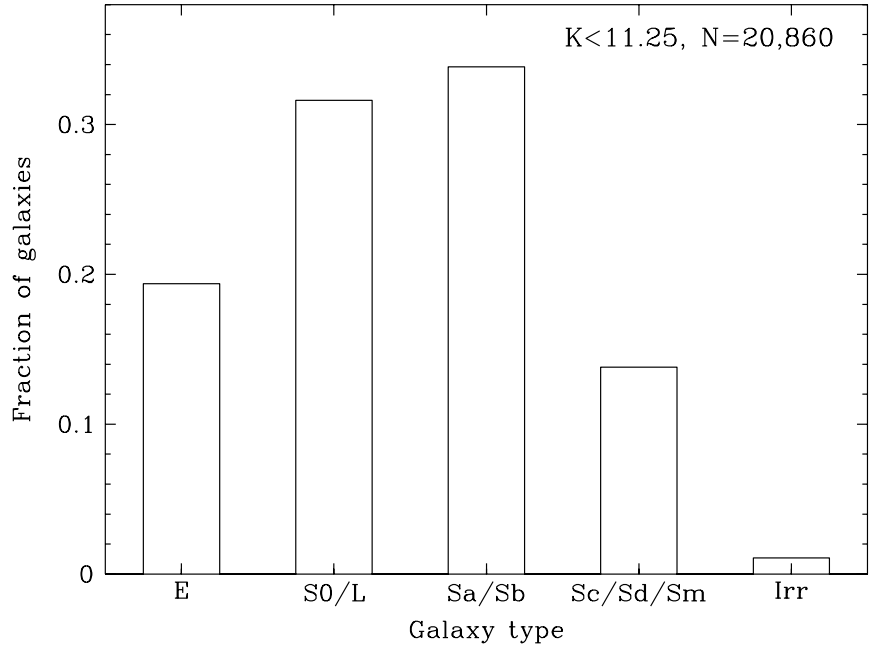

Figure 13. Histogram of the distribution of galaxy types for the $K_{s}<11.25 \mathrm{mag}$, $|b|>10^{\circ}$ sample.

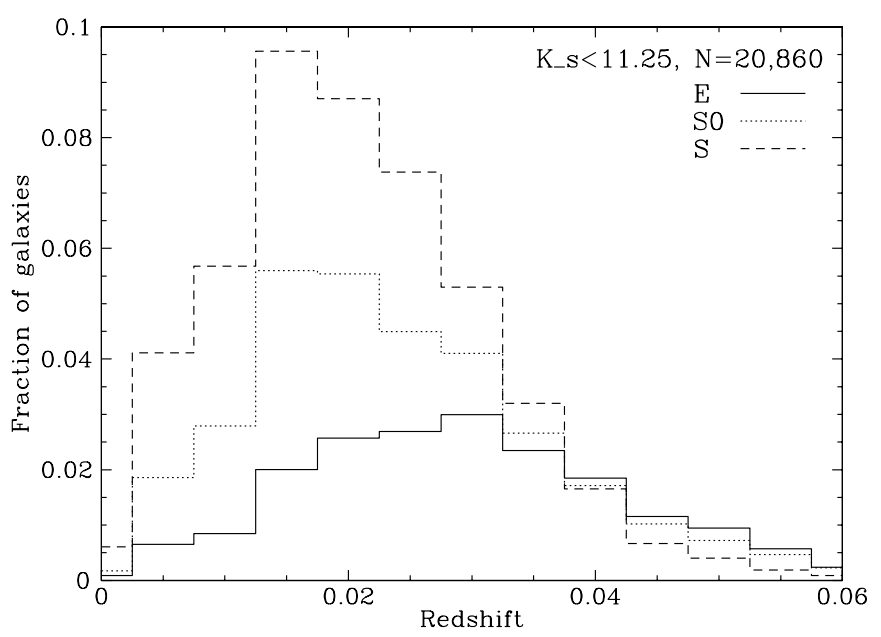

Figure 14. Histogram of $2 \mathrm{MRS} 11.25$ galaxies as a function of redshift for the three main morphological classes.

concentrations to explain the misalignment between the $\mathrm{CMB}$ velocity vector and the $2 \mathrm{MRS}$ dipole.

6. Lavaux et al. (2010) derived the peculiar velocity field for 2MRS11.25 using an orbit-reconstruction algorithm and estimated the mean matter density within $3000 \mathrm{~km} \mathrm{~s}^{-1}$ to be $\Omega_{m}=0.31 \pm 0.05$. They also studied the convergence toward the CMB dipole and found that less than half of the amplitude is generated within $40 \mathrm{~h}^{-1} \mathrm{Mpc}$.

7. Davis et al. (2011) compared 2MRS11.25 to the SFI++ peculiar velocity survey (Masters et al. 2006; Springob et al. $2007)$ to place constraints on the bias between galaxies and dark matter halos, as well as $\beta=f(\Omega) / b$ (where $f$ is the rate of growth of structure and $b$ is the bias factor) and $\sigma_{8}$ (which measures the amplitude of the linear power spectrum on the scale of $8 h^{-1} \mathrm{Mpc}$ ).

\section{SUMMARY}

2MASS has fulfilled its goal of providing an extremely uniform, deep, and unbiased survey of the nearby universe. The $2 \mathrm{MRS}$ is $97.6 \%$ complete to a limiting magnitude of $K_{s}=11.75$ mag over $91 \%$ of the sky, and its catalog contains redshifts for 43,533 galaxies.
2MRS has produced an essentially complete map of the Local Universe out to $z \sim 0.08$. While the characteristics of the structures are similar to what has been seen before, we now have a nearly full view of the nearby universe. Now we need to measure not only the redshifts, but also real distances (e.g., Masters et al. 2008) to extract the full measure of cosmological information.

This paper was written in part while J.P.H. was a Sackler visitor at the Institute of Astronomy, Cambridge, UK. We also thank the staff at the Fred. L. Whipple, Cerro Tololo, and McDonald Observatories, and the entire 2MASS team.

J.P.H., K.L.M., and A.C.C. acknowledge support by the National Science Foundation under grant AST-0406906 and by the Smithsonian Institution.

L.M.M. acknowledges support by the Smithsonian Institution Visiting Scholar program, by NASA through Hubble Fellowship Grant HST-HF-01153 from the Space Telescope Science Institute, by the National Science Foundation through a Goldberg Fellowship from the National Optical Astronomy Observatory, and by the Texas A\&M University Mitchell-Heep-Munnerlyn Endowed Career Enhancement Professorship in Physics or Astronomy.

K.L.M. acknowledges funding from the Leverhulme Trust as a 2010 Early Career Fellow and from the Peter and Patricia Gruber Foundation as the 2008 IAU Fellow.

C.M.H. was supported in part by the National Science Foundation Research Experience for Undergraduates under grant No. 9731923.

O.L. acknowledges support from a Royal Society Wolfson Research Merit Award.

J.P.H. and L.M.M. were visiting astronomers at Cerro Tololo Inter-American Observatory, operated by the Association of Universities for Research in Astronomy under contract with the National Science Foundation.

This publication has made use of the following resources.

1. Data products from the Two Micron All Sky Survey, which is a joint project of the University of Massachusetts and the Infrared Processing and Analysis Center at the California Institute of Technology, funded by the National Aeronautics and Space Administration and the National Science Foundation.

2. The NASA/IPAC Extragalactic Database (NED) which is operated by the Jet Propulsion Laboratory at the California Institute of Technology, under contract with the National Aeronautics and Space Administration.

3. The 6dF Galaxy Survey (DR3), supported by Australian Research Council Discovery Projects Grant (DP-0208876). The 6dFGS web site is http://www.aao.gov.au/local/ www/6df/.

4. The Sloan Digital Sky Survey III (DR8). Funding for SDSS-III has been provided by the Alfred P. Sloan Foundation, the Participating Institutions, the National Science Foundation, and the U.S. Department of Energy. The SDSS-III Web site is http://www.sdss3.org/. SDSS-III is managed by the Astrophysical Research Consortium for the Participating Institutions of the SDSS-III Collaboration including the University of Arizona, the Brazilian Participation Group, the Brookhaven National Laboratory, the University of Cambridge, the University of Florida, the French Participation Group, the German Participation Group, the Instituto de Astrofisica de Canarias, the Michigan State/Notre Dame/ 
JINA Participation Group, Johns Hopkins University, the Lawrence Berkeley National Laboratory, the Max Planck Institute for Astrophysics, New Mexico State University, New York University, The Ohio State University, Pennsylvania State University, the University of Portsmouth, Princeton University, the Spanish Participation Group, the University of Tokyo, the University of Utah, Vanderbilt University, the University of Virginia, the University of Washington, and Yale University.

5. The VizieR catalog access tool operated at the CDS, Strasbourg, France.

6. The Digitized Sky Surveys, produced at the Space Telescope Science Institute under U.S. Government Grant NAG W-2166. The images of these surveys are based on photographic data obtained using the Oschin Schmidt Telescope on Palomar Mountain and the UK Schmidt Telescope.

7. NASA's Astrophysics Data System at the HarvardSmithsonian Center for Astrophysics.

Typing services provided by Fang, Inc.

Facilities: FLWO:1.5m (FAST), CTIO:1.5m (RCSpec), Blanco (RCSPec), Struve (es2), HET (LRS)

\section{APPENDIX}

In this appendix, we present all-sky plots of the 2MRS data set in the equatorial coordinates in Figures 15 through 18 as well as supplementary tables 8 through 13 . Table 8 lists 324 sources of galactic origin which were removed from the catalog. T.

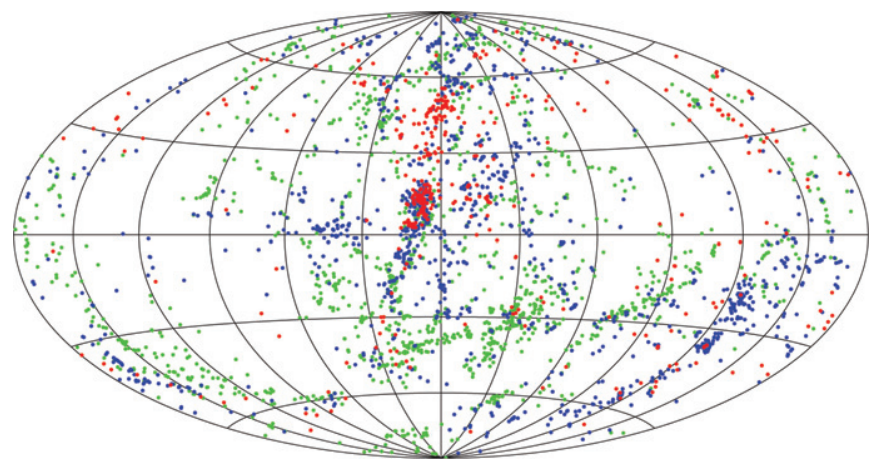

Figure 15. 2MASS galaxies inside the $3000 \mathrm{~km} \mathrm{~s}^{-1}$ sphere in equatorial coordinates (centered at R.A. $=0^{\circ}$ and following the convention of R.A. increasing to the left). Heliocentric velocities are color coded with red, blue, and green representing bins of increasing redshift/distance. Red for $V_{h}<1000 \mathrm{~km} \mathrm{~s}^{-1}$, blue for $1000<V_{h}<2000 \mathrm{~km} \mathrm{~s}^{-1}$, and green for $2000<V_{h}<3000 \mathrm{~km} \mathrm{~s}^{-1}$.

(A color version of this figure is available in the online journal.)

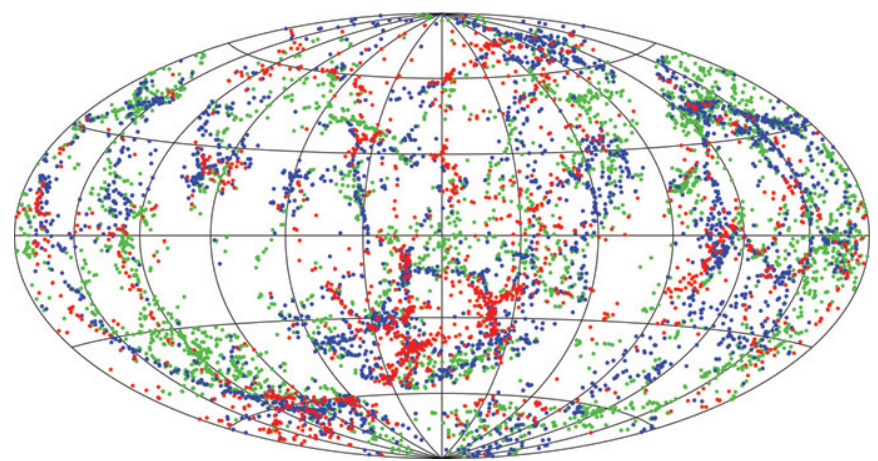

Figure 16. Same as Figure 15, but for velocities between 3000 and $6000 \mathrm{~km} \mathrm{~s}^{-1}$. (A color version of this figure is available in the online journal.)

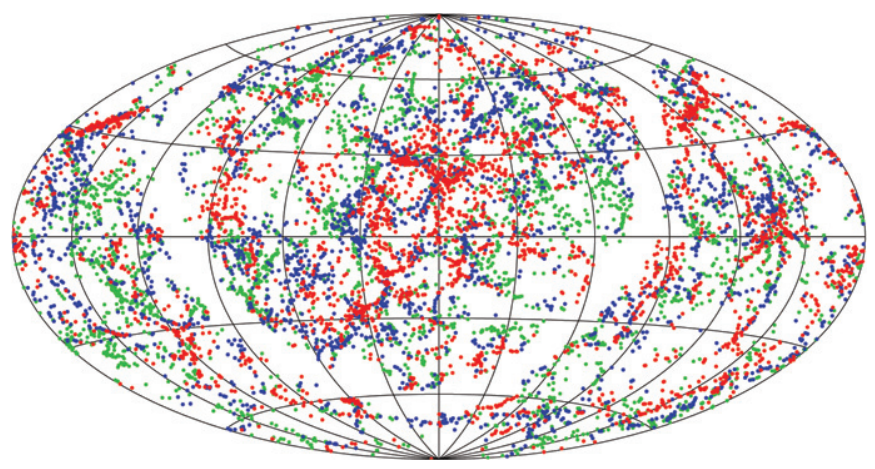

Figure 17. Same as Figure 16, but for velocities between 6000 and $9000 \mathrm{~km} \mathrm{~s}^{-1}$. (A color version of this figure is available in the online journal.)

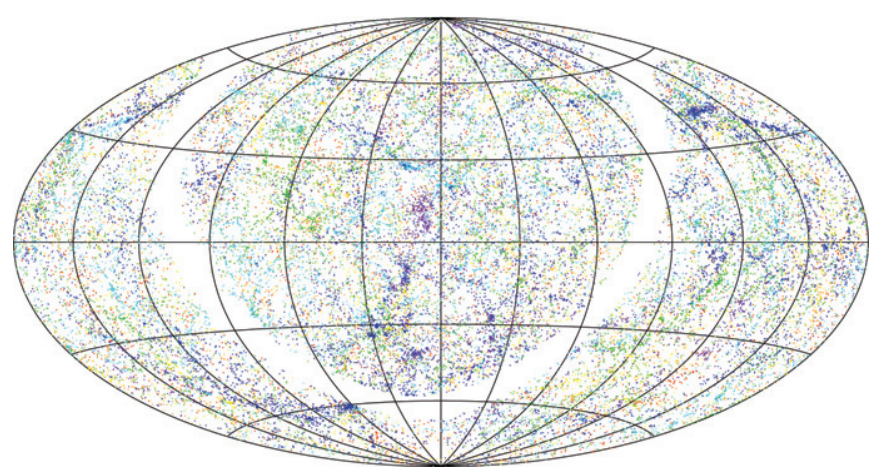

Figure 18. Same as Figure 12, but in equatorial coordinates (centered at R.A. $=0^{\circ}$ and following the convention of R.A. increasing to the left).

(A color version of this figure is available in the online journal.)

Table 8

2MASS XSC or LGA Objects Removed from Input Catalog

\begin{tabular}{ll}
\hline \hline 2MASS ID & \multicolumn{1}{c}{ Reason for Rejection } \\
\hline $00031127-5444588$ & Piece of galaxy 00031064 - 5444562 \\
$00240535-7204531$ & Globular cluster in SMC \\
$00255209-0939420$ & Piece of galaxy 00255246 - 0939427 \\
$00265282-7132113$ & Globular cluster in SMC \\
$00364578+2134078$ & Piece of galaxy 00364500+2133594 \\
$00460635-0143434$ & Piece of galaxy 00460539-0143242 \\
$00520075+6821243$ & Image flaw \\
$00523957-2637338$ & Star cluster (NGC 288) \\
$00524844-2637078$ & Star cluster (NGC 288) \\
$00525061-2635148$ & Star cluster (NGC 288) \\
$00525389-2635418$ & Star cluster (NGC 288) \\
$00584209+5628334$ & Image flaw \\
$01024864-0624482$ & Piece of galaxy 01024825 - 0624419 \\
$01081982-7252599$ & Star cluster in SMC (NGC 419) \\
$01240782-7309037$ & H II region in SMC
\end{tabular}

(This table is available in its entirety in a machine-readable form in the online journal. A portion is shown here for guidance regarding its form and content.)

Jarrett used the original 2MASS LGA pipeline to reprocess 72 of the flagged galaxies by the date this paper was submitted for publication. These galaxies are listed in Table 9. The remaining 242 flagged galaxies are separated in two categories. Table 10 lists 87 objects for which the photometric parameters are expected to exhibit little change after reprocessing, but would still benefit from such a procedure. These galaxies have 
Table 9

2MASS XSC Objects with Reprocessed Photometry

\begin{tabular}{|c|c|c|c|c|c|c|c|}
\hline \multicolumn{4}{|c|}{ Original } & \multicolumn{4}{|c|}{ Reprocessed } \\
\hline 2MASS ID & $\begin{array}{c}K_{s}^{0} \\
(\mathrm{mag})\end{array}$ & $\begin{array}{c}r_{\text {iso }} \\
\left(\log _{10} \prime\right)\end{array}$ & $b / a$ & 2MASS ID & $\begin{array}{c}K_{s}^{0} \\
(\mathrm{mag})\end{array}$ & $\begin{array}{c}r_{\text {iso }} \\
\left(\log _{10}{ }^{\prime \prime}\right)\end{array}$ & $b / a$ \\
\hline $00143065-0710028$ & 10.150 & 1.524 & 0.880 & $00143062-0710026$ & 9.976 & 1.529 & 0.810 \\
\hline $00144455-0720423$ & 8.771 & 1.721 & 0.700 & $00144460-0720430$ & 8.758 & 1.721 & 0.680 \\
\hline $00424581-2333406$ & 9.956 & 1.394 & 0.740 & $00424586-2333418$ & 9.959 & 1.338 & 0.780 \\
\hline $00510187-0703247$ & 9.317 & 1.531 & 0.400 & $00510186-0703249$ & 9.401 & 1.486 & 1.000 \\
\hline $00545028+2914482$ & 10.405 & 1.332 & 0.560 & $00545031+2914474$ & 10.392 & 1.318 & 0.570 \\
\hline $00564266-0954500$ & 9.588 & 1.695 & 0.860 & $00564267-0954507$ & 9.620 & 1.659 & 0.810 \\
\hline $01025144-6536359$ & 9.107 & 2.040 & 0.140 & $01025152-6536366$ & 9.145 & 1.989 & 0.154 \\
\hline $01243377+0143532$ & 8.801 & 1.818 & 0.980 & $01243380+0143522$ & 8.772 & 1.756 & 0.950 \\
\hline $01253143+0145335$ & 8.598 & 1.793 & 0.760 & $01253140+0145325$ & 8.692 & 1.775 & 0.710 \\
\hline $02251418-4025268$ & 10.955 & 1.243 & 0.320 & $02251422-4025268$ & 10.978 & 1.199 & 0.720 \\
\hline $02383270-0640386$ & 8.630 & 1.751 & 0.780 & $02383278-0640392$ & 8.645 & 1.718 & 0.890 \\
\hline $03011222+4454285$ & 7.721 & 1.748 & 1.000 & $03011417+4453500$ & 7.469 & 1.801 & 0.850 \\
\hline $03053084+4250076$ & 8.548 & 1.859 & 0.360 & $03053091+4250075$ & 8.525 & 1.881 & 0.380 \\
\hline $03422928-1329168$ & 8.577 & 1.989 & 0.240 & $03422931-1329174$ & 8.563 & 1.985 & 0.242 \\
\hline
\end{tabular}

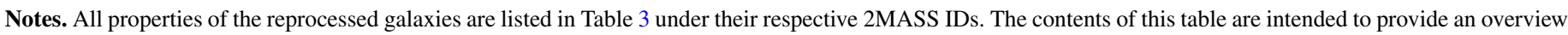
of the changes due to the reprocessed photometry.

(This table is available in its entirety in a machine-readable form in the online journal. A portion is shown here for guidance regarding its form and content.)

Table 10

2MASS XSC Galaxies with Suspect Photometry Flagged for Reprocessing at a Later Date

\begin{tabular}{l}
\hline \hline 2MASS ID \\
\hline $00364500+2133594$ \\
$01310193+4903364$ \\
$01595247-0705233$ \\
$02353199-0709366$ \\
$02352772-0921216$ \\
$02542739+4134467$ \\
$02594211+3602171$ \\
$03050558+4403167$ \\
$03235400-3730449$ \\
$03252491-1613594$ \\
$03261474+3803504$ \\
$03284660+3633226$ \\
$03392830+1323417$ \\
\hline
\end{tabular}

Notes. All properties of the flagged galaxies are listed in Table 3 under their respective 2MASS IDs. This table is only intended to provide an index of the galaxies with suspect photometry.

(This table is available in its entirety in a machine-readable form in the online journal. A portion is shown here for guidance regarding its form and content.)

not been removed from the catalog. Table 11 contains 165 galaxies with seriously compromised photometry, which have been removed from the catalog. Table 12 lists 77 galaxies for which the ZCAT and NED redshifts were in disagreement and
Table 11

2MASS XSC Galaxies with Compromised Photometry Flagged for Reprocessing and Removed from Catalog

\begin{tabular}{l}
\hline \hline 2MASS ID \\
\hline $00093966+5301008$ \\
$00112204+0623372$ \\
$00151096-2352551$ \\
$00203715+2839334$ \\
$00244982-4021070$ \\
$00271148+5103476$ \\
$00293249+5150575$ \\
$00350737+4929082$ \\
$00385467+0703458$ \\
$00452378+5353203$ \\
$00580474-8140329$ \\
$01074777+7312247$
\end{tabular}

Notes. These galaxies are not part of our catalog and therefore we do not list their photometric properties. This table is only intended to provide other users of the 2MASS $\mathrm{XSC}$ an index of galaxies that we consider to have compromised photometry.

(This table is available in its entirety in a machine-readable form in the online journal. A portion is shown here for guidance regarding its form and content.)

preference was given to the former, as well as 258 galaxies for which we assigned alternative NED redshifts. Table 13 lists additional redshifts for galaxies with multiple measurements from our survey, $6 \mathrm{dF}$ and/or SDSS. 
Table 12

Alternative Redshifts Chosen over Default NED Redshifts

\begin{tabular}{|c|c|c|c|c|c|c|c|c|c|c|}
\hline \multirow{4}{*}{$\begin{array}{l}\text { (1) } \\
\text { 2MASS ID }\end{array}$} & (2) & (3) & (4) & (5) & (6) & (7) & (8) & $(9)$ & (10) & (11) \\
\hline & \multicolumn{4}{|c|}{ NED } & \multicolumn{5}{|c|}{ Alternative } & \multirow{3}{*}{ Comments } \\
\hline & $v$ & $\sigma_{v}$ & Qual & Bibcode & $v$ & $\sigma_{v}$ & cat & sep & Bibcode & \\
\hline & \multicolumn{2}{|c|}{$\left(\mathrm{km} \mathrm{s}^{-1}\right)$} & & & \multicolumn{2}{|c|}{$\left(\mathrm{km} \mathrm{s}^{-1}\right)$} & & ' & & \\
\hline \multicolumn{11}{|c|}{ No redshift in NED for 2MASS XSC ID, but redshift exists under another ID } \\
\hline $00014401-3025082$ & ... & .. & ... & $\ldots$ & 8604 & 64 & M & 0.0 & $20032 \mathrm{dF} . . . \mathrm{C} . . .0000 \mathrm{C}$ & \\
\hline $00062997-3218179$ & $\ldots$ & $\ldots$ & $\ldots$ & $\ldots$ & 13531 & 150 & M & 0.0 & 1998MNRAS.300..417R & \\
\hline $00072080-2807072$ & $\ldots$ & $\ldots$ & $\ldots$ & $\ldots$ & 18048 & 54 & M & 0.2 & 1998AJ....116....1D & \\
\hline $00084652-3100399$ & $\ldots$ & ... & ... & $\ldots$ & 16609 & 89 & M & 0.0 & $20032 \mathrm{dF} \ldots \mathrm{C} \ldots . .0000 \mathrm{C}$ & \\
\hline $00105717-3512292$ & $\ldots$ & $\ldots$ & $\ldots$ & $\ldots$ & 14910 & 30 & $\mathrm{M}$ & 0.0 & 1986MNRAS.220..901P & \\
\hline \multicolumn{11}{|c|}{ Several discrepant redshifts in NED; alternative value given preference over default one } \\
\hline $00570857-2155112$ & 19307 & $\ldots$ & $\ldots$ & 1992NED11.R......1N & 15660 & 100 & M & 0.0 & 1978AJ....83.1549K & Only z in NED with known provenance \\
\hline $01155764+0510435$ & 5827 & 1 & $\ldots$ & 2006AJ....131..185R & 5294 & 17 & M & 0.0 & 2003AJ....126.2268W & Agrees with SAO/TDC spectral archive \#T00843 \\
\hline 01194146-3306209 & 9286 & 8 & $\ldots$ & 2003A\&A...412...57P & 5813 & 35 & M & 0.0 & 1998AJ....116...1D & Agrees with 1998MNRAS.300..417R \& 20032dF...C...0000C \\
\hline $02471331+1631590$ & 8517 & 20 & $\ldots$ & $1995 \mathrm{ApJ} . .449 . .527 \mathrm{~L}$ & 11794 & 36 & M & 0.0 & 1995ApJS..100...69F & Give preference to optical over $\mathrm{H}$ I measurement \\
\hline $03153355-1552455$ & 34674 & 39 & $\ldots$ & $1993 \mathrm{AJ} \ldots . .105 .1637 \mathrm{H}$ & 24977 & 51 & M & 0.0 & $1993 \mathrm{AJ} \ldots . .105 .1637 \mathrm{H}$ & Yields more reasonable $M_{K}$ \\
\hline \multicolumn{11}{|c|}{ Disagreement between NED and ZCAT redshifts; latter value given preference } \\
\hline $00344891+0727013$ & 555 & 10 & $\ldots$ & 1993ApJS...88..383L & 5205 & 28 & $\mathrm{O}$ & 0.0 & 20112MRS.ZMA..0000H & SAO/TDC spectral archive \#T26890 \\
\hline $00544855+1150280$ & 11425 & 408 & .. & 1995A\&AS..109..537H & 11852 & 51 & $\mathrm{O}$ & 0.0 & 20112MRS.MMT..0000H & SAO/TDC spectral archive \#M09249 \\
\hline $01193495+3210495$ & 17568 & 210 & .. & 1970PASP...82.1374v & 17902 & 40 & $\mathrm{O}$ & 0.0 & 20112MRS.JPH..0000H & \\
\hline $01465644+3206354$ & 10505 & 10 & .. & 1991RC3.9.C...0000d & 14700 & 43 & $\mathrm{O}$ & 0.0 & 20112MRS.ZMA..0000H & SAO/TDC spectral archive \#T26803 \\
\hline $01532586+7115067$ & 6595 & 300 & .. & 1996MNRAS.281..425M & 6838 & 38 & $\mathrm{O}$ & 0.0 & 20112MRS.JPH...0000H & \\
\hline
\end{tabular}

Notes. A few representative lines are provided here for guidance on its format and contents. Code for Column 8: NED position [M] atch and [O]ther sources in ZCAT.

(This table is available in its entirety in a machine-readable form in the online journal. A portion is shown here for guidance regarding its form and content.) 
Table 13

Redshifts from 6dFGS, SDSS, or NED for Galaxies also Observed by 2MRS

\begin{tabular}{|c|c|c|c|c|}
\hline 2MASS ID & $\begin{array}{c}v \\
\left(\mathrm{~km} \mathrm{~s}^{-1}\right)\end{array}$ & $\begin{array}{c}\sigma(v) \\
\left(\mathrm{km} \mathrm{s}^{-1}\right)\end{array}$ & $\begin{array}{l}\text { Vel } \\
\text { src }\end{array}$ & Bibcode \\
\hline $00010597-5359303$ & 9415 & 45 & 6 & 20096dF...C...0000J \\
\hline $00021610-2926230$ & 18334 & 45 & 6 & 20096dF...C...0000J \\
\hline $00023474-2948240$ & 17753 & 45 & 6 & 20096dF...C...0000J \\
\hline $00023794+1638377$ & 6350 & 19 & $\mathrm{~N}$ & 1999PASP..111..438F \\
\hline $00031064-5444562$ & 9790 & 45 & 6 & 20096dF...C...0000J \\
\hline $00032067-3008493$ & 20304 & 45 & 6 & 20096dF...C...0000J \\
\hline $00034964+0203594$ & 29320 & $\ldots$ & $\mathrm{N}$ & 2000ApJS..129..547B \\
\hline $00042463-5257316$ & 9851 & 45 & 6 & 20096dF...C...0000J \\
\hline $00043594-4528463$ & 11794 & 45 & 6 & 20096dF...C...0000J \\
\hline $00045169-5429144$ & 10879 & 45 & 6 & 20096dF...C...0000J \\
\hline $00053994-5349010$ & 11028 & 45 & 6 & 20096dF...C...0000J \\
\hline $00055690-1359448$ & 5730 & 45 & 6 & 20096dF...C...0000J \\
\hline $00062990-5350042$ & 10768 & 45 & 6 & 20096dF...C...0000J \\
\hline $00065396+0821027$ & 11596 & 52 & $\mathrm{~N}$ & 1999PASP..111..438F \\
\hline $00070925-2550122$ & 19118 & 45 & 6 & 20096dF...C...0000J \\
\hline
\end{tabular}

Notes. Code for Column 4: [N]ED 2MASS ID match, [S]DSS-DR8, and [6]dFGS.

(This table is available in its entirety in a machine-readable form in the online journal. A portion is shown here for guidance regarding its form and content.)

\section{REFERENCES}

Aaronson, M., Huchra, J., Mould, J., Schechter, P. L., \& Tully, R. B. 1982, ApJ, 258,64

Acker, A., Stenholm, B., \& Veron, P. 1991, A\&AS, 87, 499

Agüero, E. L., Paolantonio, S., \& Suarez, F. 1996, PASP, 108, 1117

Agüero, E. L., Suarez, F., \& Paolantonio, S. 1995, PASP, 107, 959

Aihara, H., Allende Prieto, C., An, D., et al. 2011, ApJS, 193, 29

Allen, D. A., Norris, R. P., Meadows, V. S., \& Roche, P. F. 1991, MNRAS, 248, 528

Allen, D. A., Roche, P. F., \& Norris, R. P. 1985, MNRAS, 213, 67P

Alloin, D., Barvainis, R., Gordon, M. A., \& Antonucci, R. R. J. 1992, A\&A, 265,429

Anton, K. 1993, A\&A, 270, 60

Appenzeller, I., Thiering, I., Zickgraf, F.-J., et al. 1998, ApJS, 117, 319

Arakelyan, M. A., Dibaj, E. A., \& Esipov, V. F. 1975, Astrofizika, 11, 15

Arakelyan, M. A., Dibaj, E. A., \& Esipov, V. F. 1976, Astrofizika, 12, 683

Arp, H. 1981, ApJS, 46, 75

Augarde, R., Chalabaev, A., Comte, G., Kunth, D., \& Maehara, H. 1994, A\&AS, 104, 259

Bade, N., Fink, H. H., Engels, D., Voges, W., Hagen, H.-J., Wisotzki, L., \& Reimers, D. 1995, A\&AS, 110, 469

Banks, G. D., Disney, M. J., Knezek, P. M., et al. 1999, ApJ, 524, 612

Barbon, R. 1969, Mem. Soc. Astron. Ital., 40, 559

Bardelli, S., Zucca, E., \& Baldi, A. 2001, MNRAS, 320, 387

Bardelli, S., Zucca, E., Vettolani, G., et al. 1994, MNRAS, 267, 665

Bardelli, S., Zucca, E., Zamorani, G., Moscardini, L., \& Scaramella, R. 2000, MNRAS, 312, 540

Barmby, P., \& Huchra, J. P. 1998a, AJ, 116, 1508

Barmby, P., \& Huchra, J. P. 1998b, AJ, 115, 6

Barnes, D. G., Webster, R. L., Schmidt, R. W., \& Hughes, A. 1999, MNRAS, 309,641

Barton, E. J., de Carvalho, R. R., \& Geller, M. J. 1998, AJ, 116, 1573

Bassani, L., Molina, M., Malizia, A., et al. 2006, ApJ, 636, L65

Batuski, D. J., \& Burns, J. O. 1985, ApJ, 299, 5

Batuski, D. J., Miller, C. J., Slinglend, K. A., et al. 1999, ApJ, 520, 491

Bauer, F. E., Condon, J. J., Thuan, T. X., \& Broderick, J. J. 2000, ApJS, 129, 547

Beers, T. C., Gebhardt, K., Forman, W., Huchra, J. P., \& Jones, C. 1991, AJ, 102,1581

Beers, T. C., Gebhardt, K., Huchra, J. P., et al. 1992, ApJ, 400, 410

Beers, T. C., Kriessler, J. R., Bird, C. M., \& Huchra, J. P. 1995, AJ, 109, 874

Bell, E. F., \& de Jong, R. S. 2001, ApJ, 550, 212

Bender, R., Paquet, A., \& Nieto, J.-L. 1991, A\&A, 246, 349

Benedict, F. G., Smith, B. J., \& Kenney, J. D. P. 1996, AJ, 111, 1861

Bergvall, N., Laurikainen, E., \& Aalto, S. 2003, A\&A, 405, 31

Bergwall, N. A. S., Ekman, A. B. G., Lauberts, A., et al. 1978, A\&AS, 33, 243

Bernardi, M., Alonso, M. V., da Costa, L. N., et al. 2002, AJ, 123, 2990
Berrington, R. C., Lugger, P. M., \& Cohn, H. N. 2002, AJ, 123, 2261

Bettoni, D., \& Galletta, G. 1994, A\&A, 281, 1

Bettoni, D., Moles, M., Kjærgaard, P., Fasano, G., \& Varela, J. 2006, A\&A, 452, 811

Binggeli, B., Popescu, C. C., \& Tammann, G. A. 1993, A\&AS, 98, 275

Binggeli, B., Sandage, A., \& Tammann, G. A. 1985, AJ, 90, 1681

Bird, C. M., Dickey, J. M., \& Salpeter, E. E. 1993, ApJ, 404, 81

Bischoff, K., Pietsch, W., Boller, T., et al. 1999, in Highlights in X-ray Astronomy, ed. B. Aschenbach \& M. J. Freyberg, 226

Bondi, M., Gregorini, L., Padrielli, L., \& Parma, P. 1993, A\&AS, 101, 431

Bonfanti, P., Rampazzo, R., Combes, F., Prugniel, P., \& Sulentic, J. W. 1995, A\&A, 297, 28

Borchkhadze, T. M., Breysacher, J., Laustsen, S., Schuster, H.-E., \& West, R. M. 1977, A\&AS, 30, 35

Borne, K. D., Balcells, M., Hoessel, J. G., \& McMaster, M. 1994, ApJ, 435, 79

Boselli, A., Mendes de Oliveira, C., Balkowski, C., Cayatte, V., \& Casoli, F. 1996, A\&A, 314, 738

Bosma, A., \& Freeman, K. C. 1993, AJ, 106, 1394

Bothun, G. D., \& Schombert, J. M. 1988, ApJ, 335, 617

Bottinelli, L., Durand, N., Fouque, P., et al. 1993, A\&AS, 102, 57

Bottinelli, L., Gouguenheim, L., Fouque, P., \& Paturel, G. 1990, A\&AS, 82, 391

Bowen, D. V., Blades, J. C., \& Pettini, M. 1995, ApJ, 448, 634

Braine, J., \& Dupraz, C. 1994, A\&A, 283, 407

Brinkmann, W., Siebert, J., Reich, W., et al. 1995, A\&AS, 109, 147

Brookes, M. H., Best, P. N., Peacock, J. A., Röttgering, H. J. A., \& Dunlop, J. S. 2008, MNRAS, 385, 1297

Brunzendorf, J., \& Meusinger, H. 1999, A\&AS, 139, 141

Burbidge, E. M. 1970, ApJ, 160, L33

Burbidge, E. M., \& Strittmatter, P. A. 1972, ApJ, 172, L37

Burstein, D., Davies, R. L., Dressler, A., Faber, S. M., \& Lynden-Bell, D. 1986, in NATO ASIC Proc. 180, Galaxy Distances and Deviations from Universal Expansion, ed. B. F. Madore \& R. B. Tully (Dordrecht: Reidel), 123

Buta, R., Purcell, G. B., Cobb, M. L., Crocker, D. A., Rautiainen, P., \& Salo, H. 1999, AJ, 117, 778

Cabanela, J. E., \& Aldering, G. 1998, AJ, 116, 1094

Cabanela, J. E., \& Dickey, J. M. 1999, AJ, 118, 46

Caldwell, N., \& Rose, J. A. 1997, AJ, 113, 492

Caldwell, N., Rose, J. A., \& Concannon, K. D. 2003, AJ, 125, 2891

Canzian, B., Allen, R. J., \& Tilanus, R. P. J. 1993, ApJ, 406, 457

Cappi, A., Benoist, C., da Costa, L. N., \& Maurogordato, S. 2003, A\&A, 408, 905

Cappi, A., da Costa, L. N., Benoist, C., Maurogordato, S., \& Pellegrini, P. S. 1998, AJ, 115, 2250

Carballo, R., Warwick, R. S., Barcons, X., et al. 1995, MNRAS, 277, 1312

Carilli, C. L., Wrobel, J. M., \& Ulvestad, J. S. 1998, AJ, 115, 928

Carrasco, L., Serrano, A., Tovmassian, H. M., et al. 1997, AJ, 113, 1527

Carter, D., Inglis, I., Ellis, R. S., Efstathiou, G., \& Godwin, J. G. 1985, MNRAS, 212,471 
Carter, D., Prieur, J. L., Wilkinson, A., Sparks, W. B., \& Malin, D. F. 1988 MNRAS, 235, 813

Casoli, F., Dickey, J., Kazes, I., et al. 1996, A\&AS, 116, 193

Catinella, B., Haynes, M. P., \& Giovanelli, R. 2005, AJ, 130, 1037

Cava, A., Bettoni, D., Poggianti, B. M., et al. 2009, A\&A, 495, 707

Chamaraux, P., Cayatte, V., Balkowski, C., \& Fontanelli, P. 1990, A\&A, 229, 340

16 Huchra et al

Chamaraux, P., Kazes, I., Saito, M., Yamada, T., \& Takata, T. 1995, A\&A, 299, 347

Chamaraux, P., Masnou, J.-L., Kazés, I., et al. 1999, MNRAS, 307, 236

Chapman, G. N. F., Geller, M. J., \& Huchra, J. P. 1988, AJ, 95, 999

Charmandaris, V., \& Appleton, P. N. 1996, ApJ, 460, 686

Chavushyan, V., Mujica, R., Gorshkov, A. G., et al. 2001, Astronomy Reports, 45,79

Cheng, E. S., Saulson, P. R., Wilkinson, D. T., \& Corey, B. E. 1979, ApJ, 232, L139

Chengalur, J. N., Salpeter, E. E., \& Terzian, Y. 1993, ApJ, 419, 30

Chincarini, G., \& Rood, H. J. 1971, ApJ, 168, 321

Chincarini, G., Tarenghi, M., Sol, H., et al. 1984, A\&AS, 57, 1

Colless, M., Dalton, G., Maddox, S., et al. 2001, MNRAS, 328, 1039

Colless, M., \& Hewett, P. 1987, MNRAS, 224, 453

Colless, M., Peterson, B. A., Jackson, C., et al. 2003, ArXiv Astrophysics e-prints

Collins, C. A., Guzzo, L., Nichol, R. C., \& Lumsden, S. L. 1995, MNRAS, 274, 1071

Collobert, M., Sarzi, M., Davies, R. L., Kuntschner, H., \& Colless, M. 2006, MNRAS, 370, 1213

Comte, G., Petrosian, A. R., Ohanian, G. A., \& Stepanian, J. A. 1999, Astrofizika, 42, 149

Corey, B. E., \& Wilkinson, D. T. 1976, BAAS, 8, 351

Corsini, E. M., Pizzella, A., Coccato, L., \& Bertola, F. 2003, A\&A, 408, 873

Cortese, L., Minchin, R. F., Auld, R. R., et al. 2008, MNRAS, 383, 1519

Corwin, H. G., Jr., \& Emerson, D. 1982, MNRAS, 200, 621

Couto da Silva, T. C., \& de Souza, R. E. 2006, A\&A, 457, 425

Coziol, R. 2003, MNRAS, 344, 181

Craine, E. R., \& Warner, J. W. 1976, ApJ, 206, 359

Crampton, D., Gussie, G., Cowley, A. P., \& Schmidtke, P. C. 1997, AJ, 114, 2353

Crawford, C. S., Allen, S. W., Ebeling, H., Edge, A. C., \& Fabian, A. C. 1999, MNRAS, 306, 857

Crawford, C. S., Edge, A. C., Fabian, A. C., et al. 1995, MNRAS, 274, 75

Crawford, T., Marr, J., Partridge, B., \& Strauss, M. A. 1996, ApJ, 460, 225

Crook, A. C., Huchra, J. P., Martimbeau, N., et al. 2007, ApJ, 655, 790

Crook, A. C., Silvestri, A., \& Zukin, P. 2010, MNRAS, 401, 1219

da Costa, L. N., Pellegrini, P. S., Davis, M., et al. 1991, ApJS, 75, 935

da Costa, L. N., Pellegrini, P. S., Willmer, C., et al. 1989, AJ, 97, 315

da Costa, L. N., Willmer, C. N. A., Pellegrini, P. S., et al. 1998, AJ, 116, 1

Dahlem, M., Ehle, M., Ryder, S. D., Vlajić, M., \& Haynes, R. F. 2005, A\&A, 432,475

Dale, D. A., Giovanelli, R., Haynes, M. P., Hardy, E., \& Campusano, L. E. 1999, AJ, 118, 1468

Dale, D. A., Giovanelli, R., Haynes, M. P., et al. 1997, AJ, 114, 455

Dale, D. A., Giovanelli, R., Haynes, M. P., et al. 1998, AJ, 115, 418

Dalton, G. B. 1992, A survey of rich clusters of galaxies (Boston Spa: British Library Document Supply Centre)

Dalton, G. B., Efstathiou, G., Maddox, S. J., \& Sutherland, W. J. 1994, MNRAS, 269,151

Davis, M., Huchra, J., Latham, D. W., \& Tonry, J. 1982, ApJ, 253, 423

Davis, M., Nusser, A., Masters, K. L., et al. 2011, MNRAS, 413, 2906

Davis, M., Sargent, W., \& Tonry, J. 1985, Las Campanas Survey, private communication

Davis, M., \& Strauss, M. 1991, private communication

Davoust, E., \& Considere, S. 1995, A\&AS, 110, 19

Davoust, E., \& Contini, T. 2004, A\&A, 416, 515

de Carvalho, R. R., Ribeiro, A. L. B., Capelato, H. V., \& Zepf, S. E. 1997, ApJS, 110,1

de Grijp, M. H. K., Keel, W. C., Miley, G. K., Goudfrooij, P., \& Lub, J. 1992, A\&AS, 96, 389

de Grijp, M. H. K., Miley, G. K., Lub, J., \& de Jong, T. 1985, Nature, 314, 240

de Ruiter, H. R., Parma, P., Stirpe, G. M., et al. 1998, A\&A, 339, 34

de Souza, R. E., de Mello, D. F., \& Dos Anjos, S. 1997, A\&AS, 125, 329

de Vaucouleurs, G, de Vaucouleurs, A., Corwin, H. G., Jr., et al. 1991, Third Reference Catalogue of Bright Galaxies (Berlin Heidelberg New York: Springer-Verlag)

Dekel, A., Bertschinger, E., \& Faber, S. M. 1990, ApJ, 364, 349
Della Valle, A, Mazzei, P., Bettoni, D., Aussel, H., \& de Zotti, G. 2006, A\&A, 454,453

dell'Antonio, I. P., Bothun, G. D., \& Geller, M. J. 1996, AJ, 112, 1759

dell'Antonio, I. P., Geller, M. J., \& Fabricant, D. G. 1994, AJ, 107, 427

Denicoló, G., Terlevich, R., Terlevich, E., et al. 2005, MNRAS, 356, 1440

de Lapparent, V., Geller, M. J., \& Huchra, J. P. 1986, ApJ, 302, L1

de Vaucouleurs, G. 1956, Vistas Astron., 2, 1584

de Vaucouleurs, G. 1963, ApJS, 8, 31

de Vaucouleurs, G., de Vaucouleurs, A., \& Corwin, H. G., Jr. 1976, Second

Reference Catalogue of Bright Galaxies (Austin, TX: Univ. Texas Press)

Dennefeld, M., \& Sevre, F. 1984, A\&AS, 57, 253

di Nella, H, Couch, W. J., Parker, Q. A., \& Paturel, G. 1997, MNRAS, 287, 472

di Nella, H, Couch, W. J., Paturel, G., \& Parker, Q. A. 1996a, MNRAS, 283, 367

di Nella, H., Garcia, A. M., Garnier, R., \& Paturel, G. 1995, A\&AS, 113, 151

di Nella, H, Paturel, G., \& Walsh, A. J. 1996b, A\&AS, 118, 311

Dickens, R. J., Currie, M. J., \& Lucey, J. R. 1986, MNRAS, 220, 679

Djorgovski, S., Thompson, D. J., de Carvalho, R. R., \& Mould, J. R. 1990, AJ, 100,599

Djorgovski, S. G., Thompson, D., Maxfield, L., Vigotti, M., \& Grueff, G. 1995, ApJS, 101, 255

Domingue, D. L., Keel, W. C., \& White, R. E., III 2000, ApJ, 545, 171

Donnelly, R. H., Forman, W., Jones, C., et al. 2001, ApJ, 562, 254

Donzelli, C. J., \& Pastoriza, M. G. 1997, ApJS, 111, 181

Downes, D., Solomon, P. M., \& Radford, S. J. E. 1993, ApJ, 414, L13

Dressler, A. 1991, ApJS, 75, 241

Dressler, A., \& Shectman, S. A. 1988, AJ, 95, 284

Drinkwater, M. J., Gregg, M. D., Holman, B. A., \& Brown, M. J. I. 2001, MNRAS, 326, 1076

Drinkwater, M. J., Proust, D., Parker, Q. A., Quintana, H., \& Slezak, E. 1999, Pub. Astron. Soc. Australia, 16, 113

Durret, F., Felenbok, P., Lobo, C., \& Slezak, E. 1999, A\&AS, 139, 525

2MASS Redshift Survey 17,

Dutra, C. M., Ahumada, A. V., Claría, J. J., Bica, E., \& Barbuy, B. 2003, A\&A, 408, 287

Dutra, C. M., Bica, E., Claría, J. J., Piatti, A. E., \& Ahumada, A. V. 2001, A\&A, 371,895

Eisenhardt, P. R. M., \& Lebofsky, M. J. 1987, ApJ, 316, 70

Elmegreen, B. G., Kaufman, M., Struck, C., et al. 2000, AJ, 120, 630

Epinat, B., Amram, P., Marcelin, M., et al. 2008, MNRAS, 388, 500

Erdoğdu, P., Huchra, J. P., Lahav, O., et al. 2006a, MNRAS, 368, 1515

Erdoğdu, P., \& Lahav, O. 2009, Phys. Rev. D, 80, 043005

Erdoğdu, P., Lahav, O., Huchra, J. P., et al. 2006b, MNRAS, 373, 45

Ettori, S., Guzzo, L., \& Tarenghi, M. 1995, MNRAS, 276, 689

Faber, S. M., \& Dressler, A. 1977, AJ, 82, 187

Fabricant, D. G., Kent, S. M., \& Kurtz, M. J. 1989, ApJ, 336, 77

Fairall, A. 1990, private communication

Fairall, A. P. 1979, MNRAS, 188, 349

Fairall, A. P. 1980, MNRAS, 192, 389

Fairall, A. P. 1981, MNRAS, 196, 417

Fairall, A. P. 1983, MNRAS, 203, 47

Fairall, A. P. 1984, MNRAS, 210, 69

Fairall, A. P. 1988a, MNRAS, 230, 69

Fairall, A. P. 1988b, MNRAS, 233, 691

Fairall, A. P., \& Jones, A. 1988a, Publ. Dept. Astr. Univ. Capetown, 10, 1 (1988), 10,1

Fairall, A. P., \& Jones, A. 1988b, Monthly Notes of the Astronomical Society of South Africa, 47, 25

Fairall, A. P., \& Jones, A. 1991, Publ. Dept. Astr. Univ. Capetown, 11, 1 (1991), 11,1

Fairall, A. P., Willmer, C. N. A., Calderon, J. H., et al. 1992, AJ, 103, 11

Fairall, A. P., \& Woudt, P. A. 2006, MNRAS, 366, 267

Fairall, A. P., Woudt, P. A., \& Kraan-Korteweg, R. C. 1998b, A\&AS, 127, 463

Fairall, A. P., Woudt, P. A., \& Kraan-Korteweg, R. C. 1998, A\&AS, 127, 463

Falco, E. E., Kochanek, C. S., \& Munoz, J. A. 1998, ApJ, 494, 47

Falco, E. E., Kurtz, M. J., Geller, M. J., et al. 1999, PASP, 111, 438

Fanti, R., Gioia, I., Lari, C., \& Ulrich, M. H. 1978, A\&AS, 34, 341

Few, J. M. A., Arp, H. C., \& Madore, B. F. 1982, MNRAS, 199, 633

Filippenko, A. V., \& Chornock, R. 2003, IAU Circ., 8246, 4

Fiore, F., La Franca, F., Giommi, P., et al. 1999, MNRAS, 306, L55

Firth, P., Evstigneeva, E. A., Jones, J. B., et al. 2006, MNRAS, 372, 1856

Fischer, J.-U., Hasinger, G., Schwope, A. D., et al. 1998, Astronomische Nachrichten, 319, 347

Fisher, K. B., Huchra, J. P., Strauss, M. A., et al. 1995, ApJS, 100, 69

Fleenor, M. C., Rose, J. A., Christiansen, W. A., et al. 2006, AJ, 131, 1280

Focardi, P., \& Kelm, B. 2002, A\&A, 391, 35 
Fosbury, R. A. E., Mebold, U., Goss, W. M., \& van Woerden, H. 1977, MNRAS, 179,89

Fouque, P., Durand, N., Bottinelli, L., Gouguenheim, L., \& Paturel, G. 1990, A\&AS, 86, 473

Fouque, P., Proust, D., Quintana, H., \& Ramirez, A. 1993, A\&AS, 100, 493

Freedman, W. L., Madore, B. F., Gibson, B. K., et al. 2001, ApJ, 553, 47

Freudling, W. 1995, A\&AS, 112, 429

Freudling, W., Haynes, M. P., \& Giovanelli, R. 1992, ApJS, 79, 157

Fruscione, A., \& Griffiths, R. E. 1991, ApJ, 380, L13

Gallagher, J. S., Garnavich, P. M., Caldwell, N., et al. 2008, ApJ, 685, 752

Gallego, J., Zamorano, J., Rego, M., Alonso, O., \& Vitores, A. G. 1996, A\&AS, 120,323

Galletta, G., Sage, L. J., \& Sparke, L. S. 1997, MNRAS, 284, 773

Galli, M., Cappi, A., Focardi, P., Gregorini, L., \& Vettolani, G. 1993, A\&AS, 101,259

Gallimore, J. F., Baum, S. A., O’Dea, C. P., Pedlar, A., \& Brinks, E. 1999, ApJ, 524,684

Garay, G., Mardones, D., \& Mirabel, I. F. 1993, A\&A, 277, 405

Garcia, A. M., Bottinelli, L., Garnier, R., Gouguenheim, L., \& Paturel, G. 1992, A\&AS, 96, 435

Garcia, A. M., Bottinelli, L., Garnier, R., Gouguenheim, L., \& Paturel, G. 1994 A\&AS, 107, 265

Garilli, B., Maccagni, D., \& Tarenghi, M. 1993, A\&AS, 100, 33

Garland, C. A., Williams, J. P., Pisano, D. J., et al. 2005, ApJ, 624, 714

Garrido, O., Marcelin, M., \& Amram, P. 2004, MNRAS, 349, 225

Garrido, O., Marcelin, M., Amram, P., \& Boissin, O. 2003, A\&A, 399, 51

Gavazzi, G., Bonfanti, C., Pedotti, P., Boselli, A., \& Carrasco, L. 2000, A\&AS, 146,259

Gavazzi, G., O’Neil, K., Boselli, A., \& van Driel, W. 2006, A\&A, 449, 929

Geller, M. J., Beers, T. C., Bothun, G. D., \& Huchra, J. P. 1984, AJ, 89, 319

Geller, M. J., \& Huchra, J. P. 1983, ApJS, 52, 61

Geller, M. J., Kenyon, S. J., Barton, E. J., Jarrett, T. H., \& Kewley, L. J. 2006, AJ, 132,2243

Giovanardi, C., \& Salpeter, E. E. 1985, ApJS, 58, 623

Giovanelli, R., Avera, E., \& Karachentsev, I. D. 1997, AJ, 114, 122

Giovanelli, R., \& Haynes, M. P. 1993, AJ, 105, 1271

Giovanelli, R., Haynes, M. P., Kent, B. R., et al. 2005, AJ, 130, 2613

Giovannini, G., Feretti, L., Gregorini, L., \& Parma, P. 1988, A\&A, 199, 73

Gordon, S., Koribalski, B., \& Jones, K. 2003, MNRAS, 342, 939

Graham, A. W., Colless, M. M., Busarello, G., Zaggia, S., \& Longo, G. 1998, A\&AS, 133, 325

Graham, J. A. 1978, PASP, 90, 237

Green, M. R., Godwin, J. G., \& Peach, J. V. 1988, MNRAS, 234, 1051

Gregory, S. A., \& Burns, J. O. 1982, ApJ, 255, 373

Gregory, S. A., \& Connolly, L. P. 1973, ApJ, 182, 351

Gregory, S. A., Tifft, W. G., Moody, J. W., Newberry, M. V., \& Hall, S. M. 2000, AJ, 119, 545

Grogin, N. A., \& Geller, M. J. 1999, AJ, 118, 2561

Grogin, N. A., \& Geller, M. J. 2000, AJ, 119, 32

Grogin, N. A., Geller, M. J., \& Huchra, J. P. 1998, ApJS, 119, 277

Hagiwara, Y., Klöckner, H.-R., \& Baan, W. 2004, MNRAS, 353, 1055

Hamuy, M., Phillips, M. M., Suntzeff, N. B., et al. 1996, AJ, 112, 2408

Hamwey, R. M. 1989, PhD thesis, Dartmouth College

Hasegawa, T., Wakamatsu, K.-i., Malkan, M., et al. 2000, MNRAS, 316, 326

Haynes, M. P., Giovanelli, R., Chamaraux, P., et al. 1999, AJ, 117, 2039

Haynes, M. P., Giovanelli, R., Herter, T., et al. 1997, AJ, 113, 1197

Haynes, M. P., Magri, C., Giovanelli, R., \& Starosta, B. M. 1988, AJ, 95, 607

Haynes, M. P., van Zee, L., Hogg, D. E., Roberts, M. S., \& Maddalena, R. J. 1998, AJ, 115, 62

Heidt, J., Nilsson, K., Fried, J. W., Takalo, L. O., \& Sillanp̈äa, A. 1999, A\&A, 348,113

18 Huchra et al.

Henry, J. P., Gioia, I. M., Huchra, J. P., et al. 1995, ApJ, 449, 422

Héraudeau, P., \& Simien, F. 1998, A\&AS, 133, 317

Héraudeau, P., Simien, F., Maubon, G., \& Prugniel, P. 1999, A\&AS, 136, 509

Hewitt, A., \& Burbidge, G. 1991, ApJS, 75, 297

Hickson, P., Mendes de Oliveira, C., Huchra, J. P., \& Palumbo, G. G. 1992, ApJ, 399,353

Hill, J. M., \& Oegerle, W. R. 1993, AJ, 106, 831

Hill, J. M., \& Oegerle, W. R. 1998, AJ, 116, 1529

Hintzen, P., Oegerle, W. R., \& Scott, J. S. 1978, AJ, 83, 478

Ho, L. C., \& Kim, M. 2009, ApJS, 184, 398

Hoessel, J. G., Gunn, J. E., \& Thuan, T. X. 1980, ApJ, 241, 486

Hoffman, G. L., Lewis, B. M., \& Salpeter, E. E. 1995, ApJ, 441, 28

Hopp, U., Engels, D., Green, R. F., et al. 2000, A\&AS, 142, 417

Hopp, U., Kuhn, B., Thiele, U., et al. 1995, A\&AS, 109, 537

Huchra, J., Davis, M., Latham, D., \& Tonry, J. 1983, ApJS, 52, 89
Huchra, J., Hoessel, J., \& Elias, J. 1977, AJ, 82, 674

Huchra, J., Latham, D. W., da Costa, L. N., Pellegrini, P. S., \& Willmer, C. N. A. 1993 , AJ, 105, 1637

Huchra, J. P., Geller, M. J., de Lapparent, V., \& Corwin, H. G., Jr. 1990a, ApJS, 72,433

Huchra, J., Jarrett, T., Skrutskie, M., et al. 2005, in ASP Conf. Ser. 329, Nearby Large-Scale Structures and the Zone of Avoidance, ed. A. P. Fairall \& P. A. Woudt (San Francisco, CA: ASP), 135

Huchra, J. P., Geller, M. J., \& Corwin, H. G., Jr. 1995, ApJS, 99, 391

Huchra, J. P., Henry, J. P., Postman, M., \& Geller, J. M. 1990b, ApJ, 365, 66

Huchra, J. P., Vogeley, M. S., \& Geller, M. J. 1999, ApJS, 121, 287

Huchtmeier, W. K. 1994, A\&A, 286, 389

Huchtmeier, W. K., Hopp, U., \& Kuhn, B. 1997, A\&A, 319, 67

Huchtmeier, W. K., Karachentsev, I. D., \& Karachentseva, V. E. 2001, A\&A, 377,801

Huchtmeier, W. K., Karachentsev, I. D., Karachentseva, V. E., Kudrya, Y. N., \& Mitronova, S. N. 2005, A\&A, 435, 459

Huchtmeier, W. K., \& Richter, O.-G. 1985, A\&A, 149, 118

Huchtmeier, W. K., Sage, L. J., \& Henkel, C. 1995, A\&A, 300, 675

Impey, C. D., Sprayberry, D., Irwin, M. J., \& Bothun, G. D. 1996, ApJS, 105, 209

Iwasawa, K., Ettori, S., Fabian, A. C., Edge, A. C., \& Ebeling, H. 2000, MNRAS, 313,515

Izotov, Y. I., \& Thuan, T. X. 1998, ApJ, 500, 188

Jarrett, T. 2004, PASA, 21, 396

Jarrett, T.-H., Chester, T., Cutri, R., et al. 2000a, AJ, 120, 298

Jarrett, T. H., Chester, T., Cutri, R., Schneider, S. E., \& Huchra, J. P. 2003, AJ, 125,525

Jarrett, T. H., Chester, T., Cutri, R., et al. 2000, AJ, 119, 2498

Jones, P. A., \& McAdam, W. B. 1992, ApJS, 80, 137

Jones, D. H., Read, M. A., Saunders, W., et al. 2009, MNRAS, 399, 683

Jones, D. H., Saunders, W., Colless, M., et al. 2004, MNRAS, 355, 747

Jones, D. H., Saunders, W., Read, M., \& Colless, M. 2005, PASA, 22, 277

Jore, K. P., Broeils, A. H., \& Haynes, M. P. 1996, AJ, 112, 438

Jorgensen, I., Franx, M., \& Kjaergaard, P. 1995, MNRAS, 276, 1341

Kaldare, R., Colless, M., Raychaudhury, S., \& Peterson, B. A. 2003, MNRAS, 339,652

Kamphuis, J. J., Sijbring, D., \& van Albada, T. S. 1996, A\&AS, 116, 15

Karachentsev, I., Lebedev, V., \& Shcherbanovski, A. 1985, Bulletin d'Information du Centre de Donnees Stellaires, 29, 87

Karachentsev, I. D. 1980, ApJS, 44, 137

Karachentsev, I. D., \& Karachentseva, V. E. 1981, Astrofizika, 17, 5

Karachentsev, I. D., Karachentseva, V. E., \& Huchtmeier, W. K. 2001, A\&A, 366,428

Karachentsev, I. D., Karachentseva, V. E., \& Shcherbanovskii, A. L. 1978, Pis ma Astronomicheskii Zhurnal, 4, 483

Karachentsev, I. D., \& Kopylov, A. I. 1990, MNRAS, 243, 390

Karachentsev, I. D., Pronik, V. I., \& Chuvaev, K. K. 1975, A\&A, 41, 375

Karachentseva, V. E. 1973, Astrofizicheskie Issledovaniia Izvestiya Spetsial'noj Astrofizicheskoj Observatorii, 8, 3

Katgert, P., Mazure, A., den Hartog, R., et al. 1998, A\&AS, 129, 399

Kazarian, M. A. 1987, Astrofizika, 27, 399

Kazarian, M. A., \& Kazarian, E. S. 1987, Astrofizika, 26, 5

Keel, W. C. 1996a, ApJS, 106, 27

Keel, W. C. 1996b, AJ, 111, 696

Kenney, J. D. P., Rubin, V. C., Planesas, P., \& Young, J. S. 1995, ApJ, 438, 135

Kent, B. R., Giovanelli, R., Haynes, M. P., et al. 2008, AJ, 136, 713

Kent, S. M., \& Sargent, W. L. W. 1983, AJ, 88, 697

Khachikian, E., Ter-Kazarian, G., Sargsyan, L., Yerknapetyan, K., \& Terzian, Y. 2006, MNRAS, 368, 461

Kim, D.-C., \& Sanders, D. B. 1998, ApJS, 119, 41

Kim, D.-C., Sanders, D. B., Veilleux, S., Mazzarella, J. M., \& Soifer, B. T. 1995, ApJS, 98, 129

Kirhakos, S. D., \& Steiner, J. E. 1990, AJ, 99, 1722

Kirshner, R. P., Oemler, A., Jr., \& Schechter, P. L. 1978, AJ, 83, 1549

Kirshner, R. P., Oemler, A., Jr., Schechter, P. L., \& Shectman, S. A. 1990, AJ, 100,1409

Klaas, U., \& Elsaesser, H. 1993, A\&AS, 99, 71

Kobulnicky, H. A., Dickey, J. M., Sargent, A. I., Hogg, D. E., \& Conti, P. S. 1995, AJ, 110, 116

Kochanek, C. S., Pahre, M. A., Falco, E. E., et al. 2001, ApJ, 560, 566

Koranyi, D. M., \& Geller, M. J. 2000, AJ, 119, 44

Koranyi, D. M., \& Geller, M. J. 2002, AJ, 123, 100

Koranyi, D. M., Geller, M. J., Mohr, J. J., \& Wegner, G. 1998, AJ, 116, 2108

Koribalski, B. S., Staveley-Smith, L., Kilborn, V. A., et al. 2004, AJ, 128, 16

Koulouridis, E., Plionis, M., Chavushyan, V., et al. 2006, ApJ, 639, 37

Kowalski, M. P., Ulmer, M. P., \& Hintzen, P. 1987, AJ, 93, 1350 
Kraan-Korteweg, R. C., Fairall, A. P., \& Balkowski, C. 1995, A\&A, 297, 617

Kraan-Korteweg, R. C., Henning, P. A., \& Schröder, A. C. 2002, A\&A, 391, 887

Kraan-Korteweg, R. C., \& Huchtmeier, W. K. 1992, A\&A, 266, 150

2MASS Redshift Survey 19

Krabbe, A. C., Pastoriza, M. G., Winge, C., Rodrigues, I., \& Ferreiro, D. L. 2008, MNRAS, 389, 1593

Kregel, M., van der Kruit, P. C., \& de Blok, W. J. G. 2004, MNRAS, 352, 768

Krumm, N., \& Salpeter, E. E. 1980, AJ, 85, 1312

Kunth, D., \& Sargent, W. L. W. 1979, A\&AS, 36, 259

Kurtz, M. J., \& Mink, D. J. 1998, PASP, 110, 934

Lahav, O., Brosch, N., Goldberg, E., et al. 1998, MNRAS, 299, 24

Landt, H., Padovani, P., Perlman, E. S., et al. 2001, MNRAS, 323, 757

Lara, L., Cotton, W. D., Feretti, L., et al. 2001, A\&A, 370, 409

Lauberts, A., \& Valentijn, E. A. 1989, The surface photometry catalogue of the ESO-Uppsala galaxies (Garching: European Southern Observatory)

Laurent-Muehleisen, S. A., Kollgaard, R. I., Ciardullo, R., et al. 1998, ApJS, 118,127

Lavaux, G., Tully, R. B., Mohayaee, R., \& Colombi, S. 2010, ApJ, 709, 483

Lawrence, A., Rowan-Robinson, M., Ellis, R. S., et al. 1999, MNRAS, 308, 897

Ledlow, M. J., Loken, C., Burns, J. O., Hill, J. M., \& White, R. A. 1996, AJ, 112,388

Lipovetsky, V. A. 1986, Soobshcheniya Spetsial'noj Astrofizicheskoj Observatorii, 50,12

Longhetti, M., Rampazzo, R., Bressan, A., \& Chiosi, C. 1998a, A\&AS, 130, 251

Longhetti, M., Rampazzo, R., Bressan, A., \& Chiosi, C. 1998b, A\&AS, 130, 267

Lonsdale, C. J., \& Hacking, P. B. 1989, ApJ, 339, 712

Loveday, J., Peterson, B. A., Maddox, S. J., \& Efstathiou, G. 1996, ApJS, 107, 201

Lu, N. Y., Dow, M. W., Houck, J. R., Salpeter, E. E., \& Lewis, B. M. 1990, ApJ, 357,388

Lu, N. Y., \& Freudling, W. 1995, ApJ, 449, 527

Lu, N. Y., Hoffman, G. L., Groff, T., Roos, T., \& Lamphier, C. 1993, ApJS, 88, 383

Lucey, J. R., Dickens, R. J., Mitchell, R. J., \& Dawe, J. A. 1983, MNRAS, 203, 545

Lynden-Bell, D., Faber, S. M., Burstein, D., et al. 1988, ApJ, 326, 19

Lynds, C. R. 1972, in IAU Symp. 44, External Galaxies and Quasi-Stellar Objects, ed. D. S. Evans, D. Wills, \& B. J. Wills (Cambridge: Cambridge Univ. Press), 376

Mahdavi, A., \& Geller, M. J. 2004, ApJ, 607, 202

Maia, M. A. G., Suzuki, J. A., da Costa, L. N., Willmer, C. N. A., \& Rite, C. 1996, A\&AS, 117, 487

Makarov, D. I., Burenkov, A. N., \& Tyurina, N. V. 2001, Astronomy Letters, 27,213

Makarov, D. I., Karachentsev, I. D., \& Burenkov, A. N. 2003, A\&A, 405, 951

Makarov, D. I., Karachentsev, I. D., Burennkov, A. N., Tyurina, N. V., \& Korotkova, G. G. 1997, Astronomy Letters, 23, 638

Malumuth, E. M., Kriss, G. A., Dixon, W. V. D., Ferguson, H. C., \& Ritchie, C. 1992, AJ, 104, 495

Mamon, G. A., Parker, Q. A., \& Proust, D. 2001, Pub. Astron. Soc. Australia, 18,232

Marcha, M. J. M., Browne, I. W. A., Impey, C. D., \& Smith, P. S. 1996, MNRAS, 281,425

Markaryan, B. E., Lipovetskii, V. A., \& Stepanyan, D. A. 1980, Astrophysics, 16,1

Martin, A. M., Giovanelli, R., Haynes, M. P., et al. 2009, ApJS, 183, 214

Martini, P., Mulchaey, J. S., \& Kelson, D. D. 2007, ApJ, 664, 761

Marzke, R. O., Huchra, J. P., \& Geller, M. J. 1996, AJ, 112, 1803

Masetti, N., Mason, E., Morelli, L., et al. 2008, A\&A, 482, 113

Masters, K. L., Springob, C. M., Haynes, M. P., \& Giovanelli, R. 2006, ApJ, 653,861

Masters, K. L., Springob, C. M., \& Huchra, J. P. 2008, AJ, 135, 1738

Masetti, N., Morelli, L., Palazzi, E., et al. 2006, A\&A, 459, 21

Matheson, T., \& Berlind, P. 2003, IAU Circ., 8146, 2

Matheson, T., Jha, S., Challis, P., Kirshner, R., \& Berlind, P. 2002, IAU Circ., 7916, 2

Mathewson, D. S., \& Ford, V. L. 1996, ApJS, 107, 97

Mathewson, D. S., Ford, V. L., \& Buchhorn, M. 1992, ApJS, 81, 413

Matthews, L. D., \& van Driel, W. 2000, A\&AS, 143, 421

Mauch, T., \& Sadler, E. M. 2007, MNRAS, 375, 931

Maurogordato, S., Proust, D., \& Balkowski, C. 1991, A\&A, 246, 39

Maurogordato, S., Proust, D., Cappi, A., Slezak, E., \& Martin, J. M. 1997, A\&AS, 123, 411
Maybhate, A., Goudfrooij, P., Schweizer, F., Puzia, T., \& Carter, D. 2007, AJ 134, 1729

Maza, J., \& Ruiz, M. T. 1989, ApJS, 69, 353

Mazzarella, J. M., Graham, J. R., Sanders, D. B., \& Djorgovski, S. 1993, ApJ, 409, 170

McCarthy, P. J., Kapahi, V. K., van Breugel, W., et al. 1996, ApJS, 107, 19

Mendel, J. T., Proctor, R. N., Forbes, D. A., \& Brough, S. 2008, MNRAS, 389, 749

Menzies, J. W., Coulson, I. M., \& Sargent, W. L. W. 1989, AJ, 97, 1576

Merighi, R., Basso, L., Vigotti, M., Lahulla, J. F., \& Lopez-Arroyo, M. 1991, A\&AS, 89, 225

Metcalfe, N., Fong, R., Shanks, T., \& Kilkenny, D. 1989, MNRAS, 236, 207

Michel, A., \& Huchra, J. 1988, PASP, 100, 1423

Miller, C. J., Krughoff, K. S., Batuski, D. J., \& Hill, J. M. 2002a, AJ, 124, 1918

Miller, N. A., Ledlow, M. J., Owen, F. N., \& Hill, J. M. 2002b, AJ, 123, 3018

Miller, N. A., \& Owen, F. N. 2001, ApJS, 134, 355

Misgeld, I., Mieske, S., \& Hilker, M. 2008, A\&A, 486, 697

Mohr, J. J., Geller, M. J., Fabricant, D. G., et al. 1996, ApJ, 470, 724

Mohr, J. J., \& Wegner, G. 1997, AJ, 114, 25

Monk, A. S., Penston, M. V., Pettini, M., \& Blades, J. C. 1988, MNRAS, 234, 193

Monnier Ragaigne, D, van Driel, W., O’Neil, K., et al. 2003a, A\&A, 408, 67

Monnier Ragaigne, D, van Driel, W., Schneider, S. E., Balkowski, C., \& Jarrett, T. H. 2003b, A\&A, 408, 465

Moody, J. W., \& Kirshner, R. P. 1988, AJ, 95, 1629

Moran, E. C., Halpern, J. P., \& Helfand, D. J. 1996, ApJS, 106, 341

Morganti, R., Oosterloo, T. A., Capetti, A., et al. 2003, A\&A, 399, 511

Motch, C., Guillout, P., Haberl, F., et al. 1998, A\&AS, 132, 341

Mould, J. 1991, private communication

Mould, J. R., Staveley-Smith, L., Schommer, R. A., et al. 1991, ApJ, 383, 467

Mould, J. R., Akeson, R. L., Bothun, G. D., et al. 1993, ApJ, 409, 14

Mulder, P. S., \& van Driel, W. 1993, A\&A, 272, 63

Müller, K. R., Wegner, G., Raychaudhury, S., \& Freudling, W. 1999, A\&AS, 140,327

Munn, J. A., Koo, D. C., Kron, R. G., et al. 1997, ApJS, 109, 45

Muriel, H., Nicotra, M. A., \& Lambas, D. G. 1995, AJ, 110, 1032

Nakanishi, K., Takata, T., Yamada, T., et al. 1997, ApJS, 112, 245

Neill, J. D., Brodie, J. P., Craig, W. W., Hailey, C. J., \& Misch, A. A. 2001, ApJ, 548,550

Nilsson, K., Pursimo, T., Heidt, J., et al. 2003, A\&A, 400, 95

Nishiura, S., Shimada, M., Ohyama, Y., Murayama, T., \& Taniguchi, Y. 2000, AJ, 120,1691

20 Huchra et al.

Nordgren, T. E., Chengalur, J. N., Salpeter, E. E., \& Terzian, Y. 1997b, AJ, 114, 913

Nordgren, T. E., Chengalur, J. N., Salpeter, E. E., \& Terzian, Y. 1997a, AJ, 114, 77

Norris, M. A., Sharples, R. M., \& Kuntschner, H. 2006, MNRAS, 367, 815

Oegerle, W. R., Hill, J. M., \& Fitchett, M. J. 1995, AJ, 110, 32

Ogando, R. L. C., Maia, M. A. G., Pellegrini, P. S., \& da Costa, L. N. 2008, AJ, 135,2424

Olling, R. P. 1996, AJ, 112, 457

O'Neil, K. 2004, AJ, 128, 2080

Oosterloo, T., \& Shostak, S. 1993, A\&AS, 99, 379

Osterbrock, D. E., \& De Robertis, M. M. 1985, PASP, 97, 1129

Ostriker, E. C., Huchra, J. P., Geller, M. J., \& Kurtz, M. J. 1988, AJ, 96, 1775

Owen, F. N., Ledlow, M. J., \& Keel, W. C. 1995, AJ, 109, 14

Owen, F. N., Ledlow, M. J., Morrison, G. E., \& Hill, J. M. 1997, ApJ, 488, $\mathrm{L} 15+$

Owen, F. N., White, R. A., \& Thronson, H. A., Jr. 1988, AJ, 95, 1

Padovani, P., \& Giommi, P. 1995, MNRAS, 277, 1477

Palumbo, G. G. C., Tanzella-Nitti, G., \& Vettolani, G. 1983, Catalogue of radial velocities of galaxies (New York: Gordon and Breach)

Pantoja, C. A., Altschuler, D. R., Giovanardi, C., \& Giovanelli, R. 1997, AJ, 113,905

Parker, Q. A., MacGillivray, H. T., Hill, P. W., \& Dodd, R. J. 1986, MNRAS, 220, 901

Parker, Q. A., \& Watson, F. G. 1990, A\&AS, 84, 455

Paturel, G., Theureau, G., Bottinelli, L., et al. 2003, A\&A, 412, 57

Peebles, P. J. E. 1976, ApJ, 205, 318

Pence, W. D., \& Rots, A. H. 1997, ApJ, 478, 107

Peng, B., Strom, R. G., Wei, J., \& Zhao, Y. H. 2004, A\&A, 415, 487

Penny, S. J., \& Conselice, C. J. 2008, MNRAS, 383, 247

Perlmutter, S., Aldering, G., Goldhaber, G., et al. 1999, ApJ, 517, 565

Perlman, E. S., Padovani, P., Giommi, P., et al. 1998, AJ, 115, 1253

Peterson, B. A., Ellis, R. S., Efstathiou, G., et al. 1986, MNRAS, 221, 233 
Petrosian, A., McLean, B., Allen, R. J., \& MacKenty, J. W. 2007, ApJS, 170 33

Petrosian, A. R., Saakian, K. A., \& Khachian, E. E. 1979, Astrofizika, 15, 373

Pietsch, W., Bischoff, K., Boller, T., et al. 1998, A\&A, 333, 48

Pinkney, J., Burns, J. O., Ledlow, M. J., Gómez, P. L., \& Hill, J. M. 2000, AJ, 120,2269

Pinkney, J., Rhee, G., Burns, J. O., et al. 1993, ApJ, 416, 36

Pocock, A. S., Penston, M. V., Pettini, M., \& Blades, J. C. 1984, MNRAS, 210, 373

Postman, M., Geller, M. J., \& Huchra, J. P. 1988, AJ, 95, 267

Postman, M., Huchra, J. P., \& Geller, M. J. 1986, AJ, 92, 1238

Postman, M., \& Lauer, T. R. 1995, ApJ, 440, 28

Pott, J.-U., Hartwich, M., Eckart, A., et al. 2004, A\&A, 415, 27

Poulain, P., Nieto, J.-L., \& Davoust, E. 1992, A\&AS, 95, 129

Prieur, J.-L. 1988, Etude de galaxies a Coquilles (Toulouse: Universite Sabatier, Observatoire Midi-Pyrenees)

Proust, D., Capelato, H. V., Hickel, G., et al. 2003, A\&A, 407, 31

Proust, D., Mazure, A., Vanderriest, C., Sodre, L., Jr., \& Capelato, H. V. 1995, A\&AS, 114, 565

Qiao, Q. Y., Qiu, Y. L., Li, W. D., et al. 1997, IAU Circ., 6775, 1

Quintana, H., \& de Souza, R. 1993, A\&AS, 101, 475

Quintana, H., Fouque, P., \& Way, M. J. 1994, A\&A, 283, 722

Quintana, H., Melnick, J., Proust, D., \& Infante, L. 1997, A\&AS, 125, 247

Quintana, H., \& Ramirez, A. 1990, AJ, 100, 1424

Quintana, H., \& Ramirez, A. 1995, ApJS, 96, 343

Quintana, H., Ramirez, A., Melnick, J., Raychaudhury, S., \& Slezak, E. 1995, AJ, 110, 463

Quintana, H., Ramirez, A., \& Way, M. J. 1996, AJ, 112, 36

Radburn-Smith, D. J., Lucey, J. R., Woudt, P. A., Kraan-Korteweg, R. C., \& Watson, F. G. 2006, MNRAS, 369, 1131

Ramella, M., Focardi, P., \& Geller, M. J. 1996, A\&A, 312, 745

Ramella, M., Geller, M. J., Huchra, J. P., \& Thorstensen, J. R. 1995, AJ, 109, 1458

Ramella, M., Geller, M. J., Pisani, A., \& da Costa, L. N. 2002, AJ, 123, 2976

Rampazzo, R., Reduzzi, L., Sulentic, J. W., \& Madejsky, R. 1995, A\&AS, 110, 131

Rand, R. J. 1995, AJ, 109, 2444

Ratcliffe, A., Shanks, T., Parker, Q. A., et al. 1998, MNRAS, 300, 417

Reshetnikov, V., Bournaud, F., Combes, F., Fáundez-Abans, M., \& de OliveiraAbans, M. 2006, A\&A, 446, 447

Reshetnikov, V. P., \& Combes, F. 1994, A\&A, 291, 57

Reshetnikov, V. P., Fáundez-Abans, M., \& de Oliveira-Abans, M. 2001, MNRAS, 322, 689

Rhee, G. F. R. N., \& Katgert, P. 1988, A\&AS, 72, 243

Rhee, M.-H., \& van Albada, T. S. 1996, A\&AS, 115, 407

Richter, O.-G. 1987a, A\&AS, 67, 261

Richter, O.-G. 1987b, A\&AS, 67, 237

Richter, O.-G. 1989, A\&AS, 77, 237

Richter, O.-G., \& Huchtmeier, W. K. 1987, A\&AS, 68, 427

Riess, A. G., Filippenko, A. V., Challis, P., et al. 1998, AJ, 116, 1009

Rines, K., Geller, M. J., Diaferio, A., Kurtz, M. J., \& Jarrett, T. H. 2004, AJ, 128,1078

Rines, K., Geller, M. J., Diaferio, A., et al. 2002, AJ, 124, 1266

Rines, K., Geller, M. J., Diaferio, A., Mohr, J. J., \& Wegner, G. A. 2000, AJ, 120, 2338

Rines, K., Geller, M. J., Kurtz, M. J., \& Diaferio, A. 2003, AJ, 126, 2152

Rines, K., Geller, M. J., Kurtz, M. J., et al. 2001, ApJ, 561, L41

Rodgers, A. W., Peterson, B. A., \& Harding, P. 1978, ApJ, 225, 768

Rodŕıguez-Ardila, A., Pastoriza, M. G., \& Donzelli, C. J. 2000, ApJS, 126, 63

Roman, A. T., Nakanishi, K., Tomita, A., \& Saito, M. 1996, PASJ, 48, 679

Roman, A. T., Takeuchi, T. T., Nakanishi, K., \& Saito, M. 1998, PASJ, 50, 47

Romer, A. K., Nichol, R. C., Holden, B. P., et al. 2000, ApJS, 126, 209

Rood, H. I. 1980, Catalog of galaxy redshifts (Princeton: Institute for Advanced Studies)

Rothberg, B., \& Joseph, R. D. 2006, AJ, 131, 185

Rubin, V. C., Kenney, J. D. P., \& Young, J. S. 1997, AJ, 113, 1250

Ruiz, J. R., Crenshaw, D. M., Kraemer, S. B., et al. 2005, AJ, 129, 73

Sadler, E. M., Oosterloo, T. A., Morganti, R., \& Karakas, A. 2000, AJ, 119, 1180

Saintonge, A., Giovanelli, R., Haynes, M. P., et al. 2008, AJ, 135, 588

Sakai, S., Giovanelli, R., \& Wegner, G. 1994, AJ, 108, 33

Salzer, J. J., Moody, J. W., Rosenberg, J. L., Gregory, S. A., \& Newberry, M. V. 1995, AJ, 109, 2376

Sandage, A., \& Tammann, G. A. 1981, in Carnegie Inst. of Washington Publ. 635; Vol. 0; Page 0, 0

2MASS Redshift Survey 21
Sanders, D. B., Egami, E., Lipari, S., Mirabel, I. F., \& Soifer, B. T. 1995, AJ, 110,1993

Santiago, B. X., Strauss, M. A., Lahav, O., et al. 1995, ApJ, 446, 457

Sarazin, C. L., Baum, S. A., \& O’Dea, C. P. 1995, ApJ, 451, 125

Sargent, W. L. W. 1973, ApJ, 182, L13+

Sargent, W. L. W. 1970, ApJ, 160, 405

Saunders, W., Sutherland, W. J., Maddox, S. J., et al. 2000, MNRAS, 317, 55

Sazonov, S., Revnivtsev, M., Krivonos, R., Churazov, E., \& Sunyaev, R. 2007, A\&A, 462, 57

Scarpa, R., Falomo, R., \& Pesce, J. E. 1996, A\&AS, 116, 295

Schachter, J. F., Stocke, J. T., Perlman, E., et al. 1993, ApJ, 412, 541

Schechter, P. L., \& Dressler, A. 1987, AJ, 94, 563

Schindler, S. 2000, A\&AS, 142, 433

Schmidt, B., Kirshner, R., Huchra, J., \& Brodie, J. 1993, IAU Circ., 5882, 3

Schlegel, D. J., Finkbeiner, D. P., \& Davis, M. 1998, ApJ, 500, 525

Schmoldt, I., Branchini, E., Teodoro, L., et al. 1999, MNRAS, 304, 893

Schmidt, M., \& Green, R. F. 1983, ApJ, 269, 352

Schneider, D. P., \& Gunn, J. E. 1982, ApJ, 263, 14

Schneider, S. E., Thuan, T. X., Magri, C., \& Wadiak, J. E. 1990, ApJS, 72, 245

Schneider, S. E., Thuan, T. X., Mangum, J. G., \& Miller, J. 1992, ApJS, 81, 5

Schoenmakers, A. P., de Bruyn, A. G., Röttgering, H. J. A., \& van der Laan, H. 2001, A\&A, 374, 861

Schweizer, L. Y. 1987, ApJS, 64, 411

Scodeggio, M., \& Gavazzi, G. 1993, ApJ, 409, 110

Scodeggio, M., Solanes, J. M., Giovanelli, R., \& Haynes, M. P. 1995, ApJ, 444, 41

Seeberger, R., Huchtmeier, W. K., \& Weinberger, R. 1994, A\&A, 286, 17

Seeberger, R., \& Saurer, W. 1998, A\&AS, 127, 101

Sekiguchi, K., \& Wolstencroft, R. D. 1992, MNRAS, 255, 581

Sekiguchi, K., \& Wolstencroft, R. D. 1993, MNRAS, 263, 349

Shectman, S. A., Landy, S. D., Oemler, A., et al. 1996, ApJ, 470, 172

Shier, L. M., \& Fischer, J. 1998, ApJ, 497, 163

Shostak, G. S. 1975, ApJ, 198, 527

Silk, J. 1974, ApJ, 193, 525

Simien, F., \& Prugniel, P. 1997a, A\&AS, 122, 521

Simien, F., \& Prugniel, P. 1997b, A\&AS, 126, 15

Simien, F., \& Prugniel, P. 1997c, A\&AS, 126, 519

Simien, F., \& Prugniel, P. 1998, A\&AS, 131, 287

Simien, F., \& Prugniel, P. 2000, A\&AS, 145, 263

Simien, F., \& Prugniel, P. 2002, A\&A, 384, 371

Simpson, C., Clements, D. L., Rawlings, S., \& Ward, M. 1993, MNRAS, 262, 889

Skrutskie, M. F., Cutri, R. M., Stiening, R., et al. 2006, AJ, 131, 1163

Slinglend, K., Batuski, D., Miller, C., et al. 1998, ApJS, 115, 1

Smith, B. J., Kleinmann, S. G., Huchra, J. P., \& Low, F. J. 1987, ApJ, 318, 161

Smith, R. J., Hudson, M. J., Nelan, J. E., et al. 2004, AJ, 128, 1558

Smith, R. J., Lucey, J. R., Hudson, M. J., Schlegel, D. J., \& Davies, R. L. 2000, MNRAS, 313, 469

Smith, R. J., Lucey, J. R., Steel, J., \& Hudson, M. J. 1997, MNRAS, 291, 461

Smith Castelli, A. V., Bassino, L. P., Richtler, T., et al. 2008, MNRAS, 386, 2311

Smoot, G. F., Gorenstein, M. V., \& Muller, R. A. 1977, Phys. Rev. Lett., 39, 898

Sodre, L., Jr., Capelato, H. V., Steiner, J. E., Proust, D., \& Mazure, A. 1992, MNRAS, 259, 233

Sofue, Y., \& Nakai, N. 1994, PASJ, 46, 147

Solomon, P. M., Downes, D., Radford, S. J. E., \& Barrett, J. W. 1997, ApJ, 478, 144

Spekkens, K., \& Giovanelli, R. 2006, AJ, 132, 1426

Spergel, D. N., Verde, L., Peiris, H. V., et al. 2003, ApJS, 148, 175

Springob, C. M., Masters, K. L., Haynes, M. P., Giovanelli, R., \& Marinoni, C. 2007, ApJS, 172, 599

Spinrad, H., Marr, J., Aguilar, L., \& Djorgovski, S. 1985, PASP, 97, 932

Spitzak, J. G., \& Schneider, S. E. 1998, ApJS, 119, 159

Springob, C. M., Haynes, M. P., Giovanelli, R., \& Kent, B. R. 2005, ApJS, 160, 149

Stein, P. 1996, A\&AS, 116, 203

Stein, P., Jerjen, H., \& Federspiel, M. 1997, A\&A, 327, 952

Stickel, M., \& Kuehr, H. 1993, A\&AS, 100, 395

Stocke, J. T., Keeney, B. A., Lewis, A. D., Epps, H. W., \& Schild, R. E. 2004, AJ, 127,1336

Stocke, J. T., Morris, S. L., Gioia, I. M., et al. 1991, ApJS, 76, 813

Storchi-Bergmann, T., Rodriguez-Ardila, A., Schmitt, H. R., Wilson, A. S., \& Baldwin, J. A. 1996, ApJ, 472, 83

Stockton, A. 1972, ApJ, 173, 247

Strauss, M. A., Davis, M., Yahil, A., \& Huchra, J. P. 1990, ApJ, 361, 49

Strauss, M. A., Huchra, J. P., Davis, M., et al. 1992, ApJS, 83, 29

Strauss, M. 1991, private communication 
Strauss, M. 1997, private communication

Strauss, M. A., \& Huchra, J. 1988, AJ, 95, 1602

Szomoru, A., Guhathakurta, P., van Gorkom, J. H., et al. 1994, AJ, 108, 491

Takata, T., Tomita, A., Yamada, T., et al. 1994a, PASJ, 46, 343

Takata, T., Yamada, T., Saito, M., Chamaraux, P., \& Kazes, I. 1994b, A\&AS, 104, 529

Tammann, G. A., \& Sandage, A. 1985, ApJ, 294, 81

Tanvuia, L., Kelm, B., Focardi, P., Rampazzo, R., \& Zeilinger, W. W. 2003, AJ, 126,1245

Tarenghi, M., Garilli, B., \& Maccagni, D. 1994, AJ, 107, 1629

Teague, P. F., Carter, D., \& Gray, P. M. 1990, ApJS, 72, 715

Theureau, G., Bottinelli, L., Coudreau-Durand, N., et al. 1998, A\&AS, 130, 333

Theureau, G., Coudreau, N., Hallet, N., et al. 2005, A\&A, 430, 373

Theureau, G., Hanski, M. O., Coudreau, N., Hallet, N., \& Martin, J.-M. 2007, A\&A, 465, 71

Thompson, D. J., Djorgovski, S., Vigotti, M., \& Grueff, G. 1992, ApJS, 81, 1

Thoraval, S., Boissé, P., \& Duvert, G. 1999, A\&A, 351, 1051

Thorstensen, J., Wegner, G., \& Boley, F. 2001, private communication

Thorstensen, J. R., Kurtz, M. J., Geller, M. J., Ringwald, F. A., \& Wegner, G. 1995, AJ, 109, 2368

Thorstensen, J. R., Wegner, G. A., Hamwey, R., et al. 1989, AJ, 98, 1143

Thuan, T. X., Lipovetsky, V. A., Martin, J.-M., \& Pustilnik, S. A. 1999, A\&AS, 139,1

Tifft, W. G. 1978, ApJ, 222, 54

Tifft, W. G. 1982, ApJS, 50, 319

Tifft, W. G., \& Cocke, W. J. 1988, ApJS, 67, 1

Tifft, W. G., \& Gregory, S. A. 1988, AJ, 95, 651

22 Huchra et al.

Tokarz, S. P., \& Roll, J. 1997, in ASP Conf. Ser. 125, Astronomical Data Analysis Software and Systems VI, ed. G. Hunt \& H. Payne (San Francisco, CA: ASP), 140

Tonry, J., \& Davis, M. 1979, AJ, 84, 1511

Tonry, J. L., Blakeslee, J. P., Ajhar, E. A., \& Dressler, A. 2000, ApJ, 530, 625

Tovmassian, H. M., Chavushyan, V. H., Verkhodanov, O. V., \& Tiersch, H. 1999, ApJ, 523, 87

Trager, S. C., Faber, S. M., \& Dressler, A. 2008, MNRAS, 386, 715

Trager, S. C., Faber, S. M., Worthey, G., \& Gonźalez, J. J. 2000, AJ, 119, 1645

Tripp, T. M., Lu, L., \& Savage, B. D. 1998, ApJ, 508, 200

Tritton, K. P. 1972, MNRAS, 158, 277

Tsvetkov, D. Y., \& Bartunov, O. S. 1993, Bulletin d'Information du Centre de Donnees Stellaires, 42, 17

Tully, R. B., Verheijen, M. A. W., Pierce, M. J., Huang, J.-S., \& Wainscoat, R. J. 1996, AJ, 112, 2471

Tully, R. B., \& Shaya, E. J. 1984, ApJ, 281, 31

Tustin, A. W., Geller, M. J., Kenyon, S. J., \& Diaferio, A. 2001, AJ, 122, 1289

Ulrich, M.-H. 1976, ApJ, 206, 364

Vader, J. P., \& Chaboyer, B. 1992, PASP, 104, 57

Vader, J. P., Frogel, J. A., Terndrup, D. M., \& Heisler, C. A. 1993, AJ, 106, 1743

van Driel, W, Combes, F., Arnaboldi, M., \& Sparke, L. S. 2002, A\&A, 386, 140

van Driel, W, Marcum, P., Gallagher, J. S., III, et al. 2001, A\&A, 378, 370

van Driel, W, van den Broek, A. C., \& Baan, W. 1995, ApJ, 444, 80

van Zee, L, Haynes, M. P., Salzer, J. J., \& Broeils, A. H. 1997, AJ, 113, 1618

Varela, J., Moles, M., Marquez, I., et al. 2004, A\&A, 420, 873

Veilleux, S., \& Osterbrock, D. E. 1987, ApJS, 63, 295

Verdes-Montenegro, L., Sulentic, J., Lisenfeld, U., et al. 2005, A\&A, 436, 443

Verheijen, M. A. W., \& Sancisi, R. 2001, A\&A, 370, 765

Veron-Cetty, M.-P., \& Veron, P. 1993, A\&AS, 100, 521
Veron-Cetty, M.-P., \& Veron, P. 1996, A Catalogue of quasars and active nuclei (Garching: European Southern Observatory)

Véron-Cetty, M.-P., \& Véron, P. 2001, A\&A, 374, 92

Vettolani, G., Cappi, A., Chincarini, G., et al. 1989, A\&AS, 79, 147

Vettolani, G., Chincarini, G., Scaramella, R., \& Zamorani, G. 1990, AJ, 99, 1709

Vettolani, G., Zucca, E., Merighi, R., et al. 1998, A\&AS, 130, 323

Visvanathan, N., \& van den Bergh, S. 1992, AJ, 103, 1057

Visvanathan, N., \& Yamada, T. 1996, ApJS, 107, 521

Vitores, A. G., Zamorano, J., Rego, M., Alonso, O., \& Gallego, J. 1996, A\&AS, 118,7

Warner, P. J., Riley, J. M., Eales, S. A., Downes, A. J. B., \& Baldwin, J. E. 1983 MNRAS, 204, 1279

Watson, F. G., Oates, A. P., Shanks, T., \& Hale-Sutton, D. 1991, MNRAS, 253, 222

Way, M. J., Quintana, H., Infante, L., Lambas, D. G., \& Muriel, H. 2005, AJ, 130, 2012

Wegner, G., Bernardi, M., Willmer, C. N. A., et al. 2003, AJ, 126, 2268

Wegner, G., Colless, M., Saglia, R. P., et al. 1999, MNRAS, 305, 259

Wegner, G., Haynes, M. P., \& Giovanelli, R. 1993, AJ, 105, 1251

Wegner, G., Thorstensen, J. R., Kurtz, M. J., et al. 2001, AJ, 122, 2893

Wegner, G., Thorstensen, J. R., Kurtz, M. J., Geller, M. J., \& Huchra, J. P. 1990, AJ, 100, 1405

Werner, P. N., Worrall, D. M., \& Birkinshaw, M. 1999, MNRAS, 307, 722

Werner, P. N., Worrall, D. M., \& Birkinshaw, M. 2000, MNRAS, 317, 105

West, R. M. 1976, A\&A, 53, 435

West, R. M., Surdej, J., Schuster, H.-E., et al. 1981, A\&AS, 46, 57

Westover, M. 2007, PhD thesis, Harvard Univ.

White, R. A., Bliton, M., Bhavsar, S. P., et al. 1999, AJ, 118, 2014

Whiteoak, J. B. 1972, Aust. J. Phys., 25, 233

Willick, J. A., Courteau, S., Faber, S. M., et al. 1997, ApJS, 109, 333

Willick, J. A., Bowyer, S., \& Brodie, J. P. 1990, ApJ, 355, 393

Willmer, C. N. A., Focardi, P., Chan, R., Pellegrini, P. S., \& da Costa, N. L. 1991, AJ, 101, 57

Wills, D., \& Wills, B. J. 1976, ApJS, 31, 143

Winkler, H. 1988, MNRAS, 234, 703

Winkler, H. 1997, MNRAS, 292, 273

Winkler, H., Stirpe, G. M., \& Sekiguchi, K. 1992, A\&AS, 94, 103

Wong, O. I., Ryan-Weber, E. V., Garcia-Appadoo, D. A., et al. 2006, MNRAS, 371,1855

Woods, D. F., Geller, M. J., \& Barton, E. J 2006, AJ, 132, 197

Woudt, P. A., Kraan-Korteweg, R. C., Cayatte, V., Balkowski, C., \& Felenbok, P. 2004, A\&A, 415, 9

Woudt, P. A., Kraan-Korteweg, R. C., \& Fairall, A. P. 1999, A\&A, 352, 39

Woudt, P. A., Kraan-Korteweg, R. C., Lucey, J., Fairall, A. P., \& Moore, S. A. W. 2008, MNRAS, 383, 445

Yamada, T., \& Saito, M. 1993, PASJ, 45, 25

Yamada, T., Tomita, A., Saito, M., Chamaraux, P., \& Kazes, I. 1994, MNRAS, 270, 93

York, D. G., Adelman, J., Anderson, J. E., Jr., et al. 2000, AJ, 120, 1579

Zabludoff, A. I., Geller, M. J., Huchra, J. P., \& Vogeley, M. S. 1993, AJ, 106, 1273

Zabludoff, A. I., Huchra, J. P., \& Geller, M. J. 1990, ApJS, 74, 1

Zabludoff, A. I., \& Mulchaey, J. S. 1998, ApJ, 496, 39

Zwaan, M. A., Briggs, F. H., Sprayberry, D., \& Sorar, E. 1997, ApJ, 490, 173

Zaroubi, S., Hoffman, Y., Fisher, K. B., \& Lahav, O. 1995, ApJ, 449, 446

Zwicky, F., \& Zwicky, M. A. 1971, Catalogue of Selected Compact Galaxies and of Post-eruptive Galaxies (Guemligen: Zwicky) 